\title{
MedienPädagogik
}

Zeitschrift für Theorie und Praxis der Medienbildung

Lernsituationen mit Metaphern und Wikibooks.

Fallstudien zu Entwicklungspotenzialen einer integrativen Medienbildung in der Lehrerinnen- und Lehrerbildung

\section{Erste Erkenntnisse und Modifikation des Entwurfs}

Franco Rau

\section{Zusammenfassung}

Der fünfte Teil der Arbeit bündelt und diskutiert zunächst die empirischen Erkenntnisse der ersten Erprobung (Kap. 8) und skizziert darauf aufbauend die entwickelten Modifikationen der Lernsituationen sowie des gesamten Seminarkonzeptes (Kap. 9). Dafür erfolgt die Interpretation der empirischen Ergebnisse auf mikrodidaktischer Ebene mit zwei Schwerpunkten: (1.) Metaphern als Artikulations- und Reflexionsanlässe (Kap. 8.2) und (2.) die Mitgestaltung eines Wikibooks als Erfahrungs- und Reflexionsmöglichkeit zur medienpädagogischen Professionalisierung (Kap. 8.3). Zudem erfolgt eine Diskussion der Geltungsbegründung der gewonnenen Erkenntnisse unter Berücksichtigung forschungsmethodischer Gütekriterien (Kap. 8.4). Zur Modifikation des Entwurfes werden ausgewählte Lernsituationen auf Basis der gewonnen Erkenntnisse weiterentwickelt (Kap. 9). Es erfolgt die Darstellung didaktischer Veränderungen hinsichtlich drei exemplarisch ausgewählter Lernsituationen: Visualisierung von Metaphern als neue Reflexionsmöglichkeit (Kap. 9.2.1), Modus der Gruppenbildung als Mitgestaltungsmöglichkeit (Kap. 9.2.2) und Wikibooks als offenes und veränderbares Lehr- und Lernmaterial (Kap. 9.2.3). Abschliessend wird die Modifikation des Phasenkonzeptes skizziert (Kap. 9.3) und eine Zusammenfassung des Kapitels formuliert (Kap. 9.4).

\section{Auswertung und Neuperspektivierung}

\subsection{Methodische Verortung}

Die dritte Phase einer entwicklungsorientierten Bildungsforschung bezeichnen Sesink und Reinmann (2015) als "Auswertung und Neuperspektivierung» (Kap. 3.1.3). Diese Phase umfasst zwei zentrale Auswertungs- bzw. Interpretationsrichtungen. Zum einen geht es um die kritische Prüfung der Frage, «ob das eingetreten ist, was der Entwurf vorgesehen hatte» (Sesink und Reinmann 2015, 80). Zum anderen geht es um die zukunftsgerichtete Interpretation der Ergebnisse, welche die Basis für einen modifizierten Konzeptentwurf darstellt (Abb. 8.1). Das vorliegende Kapitel widmet sich diesen zwei zentralen Auswertungs- bzw. Interpretationsrichtungen für die im Sommersemester 2015 durchgeführten Erprobungen und Analysen (Kap. 7). Die Bearbeitung der ersten Interpretationsrichtung umfasst den kontinuierlichen Vergleich der Ergebnisse der experimentellen Praxis (Kap. 7.4) mit den im Entwurf 
formulierten Zielstellungen (Kap. 6). Dafür wird zwischen den zwei Zieldimensionen «pädagogische Artikulations- und Reflexionsfähigkeit» (Kap. 6.2.1) und «Teilhabe an einer partizipativen Medienkultur» (Kap. 6.2.2) unterschieden. Die zukunftsgerichtete Interpretation der Ergebnisse erfolgt insbesondere zur Identifikation neuer Handlungsspielräume in der Praxis und bildet die Grundlage zur Weiterentwicklung des ersten Entwurfs. Diesen Übergang der unterschiedlichen Phasen beschreiben Sesink und Reinmann $(2015,82)$ wie folgt: "Die Auswertungsphase mündet $[\ldots]$ in den Einstieg zu einem modifizierten Entwurf, der sich als Fortentwicklung des vorhergehenden Entwurfs auf der Basis der Projekterfahrungen versteht». Zur Darstellung dieses «fliessenden» Übergangs werden im Rahmen des folgenden Kapitels bereits Hinweise und Verweise auf die vorgenommene Entwurfsmodifikation gegeben (Kap. 9).

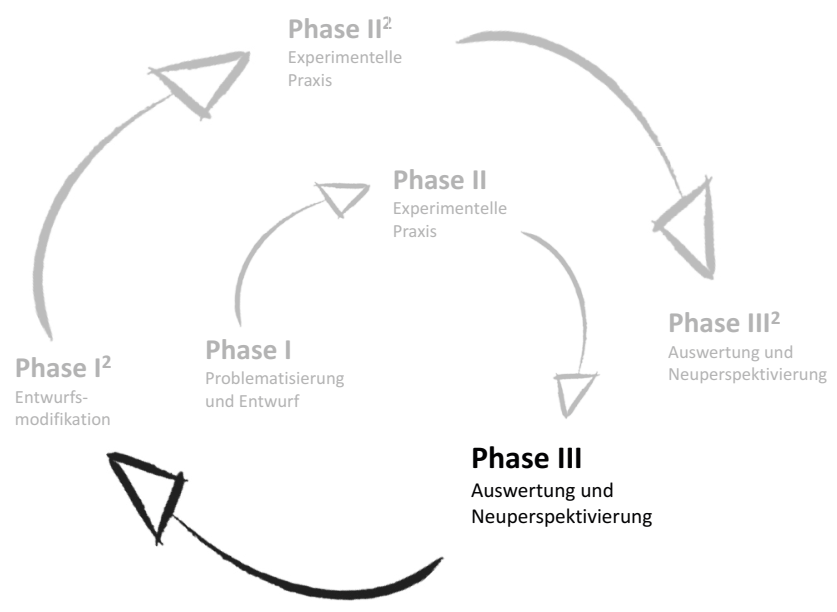

Abb. 8.1: Verortung des Kapitels im Phasenmodell der entwicklungsorientierten Bildungsforschung.

Forschungsmethodisch kann das Vorgehen als vergleichende Betrachtung und Interpretation der verschiedenen Datenerhebungen und -analysen der experimentellen Praxis (Kap. 7) beschrieben werden. Dies erfolgt in einer systematischen Triangulation unterschiedlicher Perspektiven: der Perspektive der Studierenden, der Perspektive des Lehrenden sowie eine objektorientierte Perspektive (Kap. 3.2.2). Im Anschluss erfolgt - wie von Sesink und Reinmann (2015) gefordert - eine Diskussion der Geltungsgrenzen der Ergebnisse sowie eine kritische Betrachtung der forschungsmethodisch angemessenen Durchführung der verschiedenen Verfahren (Kap. 8.4). Zur kriteriengeleiteten Betrachtung der empirischen Ergebnisse werden Gütekriterien qualitativer Forschung in Anlehnung an Steinke (2012) und Flick (2012) verwendet.

Das beschriebene Vorgehen erfüllt im Sinne der von Tulodziecki et al. $(2013,229)$ vorgeschlagenen Prinzipien gestaltungs- und entwicklungsorientierter Bildungsforschung u. a. zwei Funktionen (Kap. 2.2.1). Zum einen erfolgt im Rahmen des Kapitels 
die Berücksichtigung des Prozessstandards zur «Verknüpfung unterschiedlicher Vorgehensweisen bei der Datenerfassung und Auswertung» (Tulodziecki et al. 2013, 229) hinsichtlich der formulierten Fragestellung. Zum anderen erfolgt die Reflexion bzw. das «Einhalten von Gütekriterien und Qualitätsstandards» (Tulodziecki et al. 2013, 230) in Bezug auf die Durchführung der empirischen Forschungsmethoden.

\subsection{Metaphern als Artikulations- und Reflexionsanlässe}

Die (Weiter-)Entwicklung pädagogischer Artikulations- und Reflexionsfähigkeit seitens der Studierenden wurde als eine übergeordnete Zielstellung im Rahmen des Entwurfs formuliert (Kap. 6.2.1). Eine notwendige Bedingung zur Weiterentwicklung dieser Fähigkeit wird in der Auseinandersetzung mit den eigenen erfahrungsbasierten Vorstellungen gesehen. Wenn sich Studierende ihrer eigenen Vorstellungen und Überzeugungen zum Lehren und Lernen bewusstwerden, werden diese für individuelle und gemeinsame Reflexionen zugänglich. In der Folge lassen sich die Vorstellungen in Relation zu pädagogischen und didaktischen Perspektiven setzen. Diese Annahme wurde im Rahmen der Problematisierung (Kap. 5.2) sowie in der Diskussion theoretischer Konstrukte (Kap. 5.3.4) diskutiert und begründet.

Die Aufgabenstellung, die eigenen Vorstellungen zum Lehren und Lernen im Unterricht in Form einer Ad-hoc-Metapher zum Ausdruck zu bringen, wurde mit der Absicht gestellt, dass sich Studierende mit ihren eigenen Vorstellungen und Überzeugen auseinandersetzen. Die Analyse der studentischen Bearbeitungen dieser Aufgabe lassen zwar keine unmittelbaren Rückschlüsse auf die Vorstellungswelt der Studierenden zu, offenbaren jedoch, wie sich Studierende im Rahmen entsprechender Anforderungssituationen artikulieren (können). Auf Basis der dargestellten Ergebnisse der Metaphernanalyse (Kap. 7.4.3), der reflektierten Praxiserfahrungen (Kap. 7.2) sowie der Interviews (Kap. 7.4.1) wird im Folgenden argumentiert, dass

- die Formulierung von Ad-hoc-Metaphern einen herausfordernden Anlass darstellt, das eigene Denken über Lehren und Lernen zum Ausdruck zu bringen (Kap. 8.2.1),

- die formulierten Ad-hoc-Metaphern vielfältige Diskussions- und Reflexionsanlässe für Einzel- und Gruppenarbeiten bieten können (Kap. 8.2.2),

- die Erarbeitung einer Gruppenmetapher in Anlehnung und als Ausdifferenzierung ausgewählter Ad-hoc-Metaphern erfolgen kann (Kap. 8.2.3)

- eine mediengestalterische Perspektive zur Visualisierung der sprachlich verfassten Metaphern weitere Reflexionsanlässe eröffnen kann (Kap. 8.2.4). 


\subsubsection{Ad-hoc-Metaphern als herausfordernder Anlass und Ausdrucksmöglichkeit}

Ad-hoc-Metaphern bieten einen herausfordernden Artikulationsanlass sowie die Chance, dass sich Studierende den eigenen Vorstellungen und Überzeugungen zum Lehren und Lernen in der Schule bewusster werden. Die Chance wird darin gesehen, dass Studierende bei der Entwicklung und Erläuterung einer Metapher während der Ad-hoc-Situation des Seminars verschiedene - bewusste oder unbewusste - Entscheidungen treffen müssen. Zu diesen Entscheidungen gehören die Auswahl der Elemente des Zielbereichs des Lehrens und Lernens, die Auswahl eines angemessenen Bildbereichs zur Übertragung sowie Überlegungen zur nachvollziehbaren Erläuterung bzw. Darstellung der eigenen Verknüpfung zwischen Bild- und Zielbereich. Auf Basis der Erkenntnisse der Metaphernanalyse der studentischen Arbeitsprodukte (Kap. 7.4) wird im Folgenden argumentiert, dass

- es der Mehrheit der Studierenden gelang, unterschiedliche Aspekte des Lehrens und Lernens mit Hilfe eines vielfältigen Spektrums metaphorischer Ausdrucksweisen ${ }^{247}$ nachvollziehbar zu artikulieren (Kap. 7.4.3.1, 7.4.3.2),

- der Entwurf und die Formulierung von Metaphern zur Artikulation der eigenen Vorstellungen über Lehren und Lernen eine Herausforderung für Studierende darstellt. Diese Herausforderung wird insbesondere durch die sprachlichen Phänomene sichtbar, welche eine intersubjektive Nachvollziehbarkeit einzelner Ad-hocMetaphern einschränken (Kap. 7.4.3.1).

Die Verschriftlichung von (eigenen) Vorstellungen ${ }^{248}$ zum Lehren und Lernen in der Schule eröffnet die Chance, das eigene Denken sichtbar und so für Rückmeldungen sowie Diskussionen zugänglich zu machen. Die rekonstruierten metaphorischen Muster und Sprachfiguren der Studierenden (Kap. 7.4.3.2) stellen Indikatoren dafür dar, dass die Formulierung von Ad-hoc-Metapher geeignet ist, die aus der Perspektive der Studierenden relevanten Aspekte des Lehrens und Lernen zu artikulieren. So kann zum einen der Anspruch berücksichtigt werden, die Themen der Studierenden als Lernvoraussetzungen zu berücksichtigen (Kap. 6.3.1). Zum anderen wird es möglich, Studierenden Gelegenheiten zu bieten, über die eigenen Vorstellungen und Überzeugungen nachzudenken und diese zum Ausdruck zu bringen.

Die Herausforderung in der Bearbeitung der Aufgabe zeigt sich insbesondere in den beschriebenen Formen und Phänomenen, welche die intersubjektive Nachvollziehbarkeit der entwickelten Ad-hoc-Metaphern einschränken: (a) Routinisierte Metaphern «fremder» Bildbereiche (b) Bildbrüche innerhalb des Bildbereichs einer Adhoc-Metapher, (c) Nicht nachvollziehbare Bedeutungsübertragungen (Kap. 7.4.3.1).

247 Die Studierenden verwendeten u. a. Bildbereiche des Pflanzens und Wachsen, der Fortbewegung, des Bauens, des Handelns etc. (Kap. 7.4.3.2).

248 Inwiefern diese Entscheidungen und Reflexion der eigenen Vorstellungen den Studierenden bereits vor der Formulierung bewusst waren oder durch die Entwicklung einer Metapher bewusstwurden, kann auf Basis der Daten nicht beurteilt werden. 
Aufgrund der Durchdringung unserer Alltags- und pädagogischen Fachsprache mit vielfältigen Metaphern (z. B. Kap. 5.5.1, 5.4.1) ist die Verwendung routinisierter Metaphern einerseits nicht überraschend. Das Phänomen kann aber anderseits als Indikator dafür gedeutet werden, dass sich Studierende der Metaphorik unserer Sprache über erziehungswissenschaftliche und -praktische Zusammenhänge nicht bewusst sind. Ferner erscheint es möglich, dass nicht alle Studierenden die Aufgabenstellung zur Formulierung einer Ad-hoc-Metapher als sinnvollen Artikulations- und Reflexionsanlass erlebten und mithilfe defensiver Lernstrategien (Grell 2006; Holzkamp 1995) versuchten, die Aufgabe zu bewältigen.

\subsubsection{Potenzielle Diskussions- und Reflexionsanlässe der Ad-hoc-Metaphern}

Über die Artikulation einer eigenen Ad-hoc-Metapher hinaus (Kap. 8.2.1) wird im Folgenden argumentiert, dass die Auseinandersetzung mit fremden Ad-hoc-Metaphern potenzielle Diskussions- und Reflexionsanlässe eröffnet. Die Aufgabenstellungen zur Formulierung von Ad-hoc-Metaphern führte zu vielfältigen Ausdrucksweisen zum Lehren und Lernen in der Schule. Diese Vielfalt zeigte sich in den unterschiedlichen Sprachfiguren und metaphorischen Mustern (Kap. 7.4.3.2). Bei vergleichender Betrachtung dieser Sprachfiguren und Muster lässt sich zeigen, dass sich diese zu einer reflexiven Auseinandersetzung mit didaktischen Fragestellungen eignen. Dies erfolgt exemplarisch hinsichtlich der folgenden vier Aspekte:

- Rollenbilder von Lehrenden und Lernenden (Kap. 8.2.2.1)

- Bedeutung von Methoden und Medien für das Lehren und Lernen (Kap. 8.2.2.2)

- Gendergerechte Sprache der Metaphern (Kap. 8.2.2.3)

- Möglichkeiten und Grenzen metaphorischer Vergleiche (Kap. 8.2.2.4)

Ausgehend von studentischen Arbeitsprodukten wird an exemplarisch ausgewählten Aspekten gezeigt, wie eine diskursive und vergleichende Auseinandersetzung mit den Sprachfiguren und metaphorischen Mustern potenzielle Anlässe zur Selbstreflexion, zur Bewusstwerdung eigener Vorstellungen und Sprachfiguren sowie zur Reflexion didaktischer Fragestellungen eröffnen kann. Inwiefern diese potenziellen Anlässe im Rahmen der Erprobung wahrgenommen wurden, wird abschliessend diskutiert.

\subsubsection{Rollenbilder von Lehrenden und Lernenden}

In der Darstellung der metaphorischen Muster und Sprachfiguren der studentischen Ad-hoc-Metaphern (Kap. 7.4.3.2) wurden sehr unterschiedliche Rollenbilder von Lehrenden und Lernenden zum Ausdruck gebracht. Die Rollenbilder sowie die damit verbundenen Annahmen über relevante Aufgaben von Lehrkräften sowie von 
Schülerinnen und Schülern erscheinen teilweise als konkurrierend bzw. widersprechend und teilweise einander ergänzend. Diese Differenzen sowie damit verbundene Konsequenzen für das Denken und Sprechen über Lehren und Lernen werden im Vergleich der drei folgenden metaphorischen Muster sichtbar: «Lehren und Lernen ist wie GARTENARBEIT UND WACHSTUM», «Lehren und Lernen ist wie GEBEN UND NEHMEN» und «Lehren ist wie MATERIALBEARBEITUNG UND OBJEKTBEWEGUNG» (Kap. 7.4.3.2). In Anlehnung an Klingberg (1990) lässt sich diskutieren, inwiefern Lehrende und Lernende jeweils als Subjekt und/oder Objekt spezifischer Lehr- und Lernhandlungen beschrieben werden.

Im metaphorischen Muster «Lehren und Lernen ist wie GARTENARBEIT UND WACHSTUM» werden Lehrende als diejenigen dargestellt, die über die Gestaltung von Rahmenbedingungen Möglichkeiten schaffen, damit sich die Lernenden als Pflanzen «gut entwickeln» und «wachsen [...] können» (D15-01). Zentral an diesem Muster ist, dass die Tätigkeiten des Lehrenden nicht direkt auf die Veränderung der Pflanzen bzw. Lernenden zielen. Deutlich anders zeigt sich das Rollenbild der Lehrenden im metaphorischen Muster «Lehren als MATERIALBEARBEITUNG UND OBJEKTBEWEGUNG». Schülerinnen und Schüler scheinen hier selbst das (Roh-)Material bzw. konkrete Objekte darzustellen. Lehrkräfte können dieses Material und diese Objekte nach ihren Vorstellungen verbessern und unmittelbar auf diese einwirken. Lehrende werden - trotz unterschiedlicher Handlungsmöglichkeiten - in beiden metaphorischen Mustern als Subjekte des Lehrens dargestellt. Lernende hingegen sind nicht Subjekte des Lernens, sondern vielmehr Objekte des Lehrens. So werden Lernende in Form von Pflanzen oder Rohmaterialien und Objekten als weitgehend passiv bzw. kaum interaktiv dargestellt. Lernen ist etwas, das den Schülerinnen und Schülern passiert, ohne dass sie selbst darauf Einfluss nehmen können oder mitbestimmen könnten, was und wie sie etwas lernen.

Im Gegensatz zu diesen konstruierten Rollenbildern steht das Muster «Lehren und Lernen ist wie GEBEN UND NEHMEN» bzw. die Ad-hoc-Metapher «zum Bäcker gehen, was einzukaufen und zu essen» (D15-20). Bei dieser Metapher kann der Lernende als Subjekt seines Lernprozesses verstanden werden. Dies zeigt sich insbesondere darin, welche Handlungsoptionen und Aufgaben den Lehrenden und Lernenden zugeschrieben werden. Der "Lernende» wählt als «Käufer» aus und «entscheidet selbst, was von dem Angebot er an- und schließlich auch aufnimmt» (D15-20). Lernende haben in dieser Ad-hoc-Metapher genau jene Möglichkeiten, die ihnen im Vergleich mit einer Pflanze oder eines abstrakten Objektes nicht zugesprochen werden. Zugleich bleibt der «Lehrende» als «Verkäufer» auch Subjekt des Lehrens, wie sich an den folgenden Formulierungen erkennen lässt: «Gibt Angebot/Thema, kennt sich aus, macht schmackhaft» (D15-20). 
In dieser Differenz wird ein Irritations- und Reflexionspotenzial gesehen, welche Möglichkeiten zur Diskussion pädagogischer Perspektiven eröffnen. Mit den jeweils unterschiedlich skizzierten Rollenbildern für Lehrkräfte sowie Schülerinnen und Schülern ändert sich auch das Verständnis vom Lehren und Lernen sowie die Konzeption der Aufgaben und Handlungsmöglichkeiten der entsprechenden Personen. Durch den Vergleich der metaphorischen Konzepte sowie der verschiedenen Bildbereiche werden diese Unterschiede sichtbar und beispielsweise hinsichtlich der folgenden Fragen diskutierbar:

- In welcher Metaphorik beschreiben die Studierenden ihr eigenes Studium bzw. ihre Erfahrungen in der Schule?

- In welchen Situationen bzw. in welchem Kontext sind die verschiedenen Rollenbilder sowie die damit verbundenen Aufgaben für Lehrerinnen und Lehrer sowie Schülerinnen und Schüler argumentativ vertretbar?

- Welche Rollenbilder sind aus pädagogischer sowie didaktischer Perspektive sinnvoll und begründet?

Während die erste Frage der Selbstreflexion und Aufarbeitung der eigenen Erfahrungen dienen kann, zielt die zweite Frage auf eine Bewertung der unterschiedlichen Rollenbilder hinsichtlich spezifischer Situationen und Kontexte. Die letzte Frage eröffnet die Möglichkeit, die eigenen Rollenbilder in Relation zu pädagogischen sowie didaktischen Perspektiven zu setzen. Das Wissen über didaktische und pädagogische Grundbegriffe und Modelle kann eine Anregung bieten und neue Perspektiven eröffnen, die eigenen Metaphern oder Vorstellungen zu reflektieren Der Bezug auf eine pädagogische oder didaktische Perspektive scheint insbesondere dann hilfreich, wenn zwei gegensätzliche Überzeugungen zu keiner gemeinsamen Bewertung eines Rollenbildes gelangen. Die dritte Frage eröffnet die Möglichkeit, die verschiedenen Rollenbilder aus einer anderen Perspektive betrachten zu können bzw. betrachten zu müssen.

\subsubsection{Bedeutung von Methoden und Medien für das Lehren und Lernen}

Weitere Diskussions- und Reflexionsanlässe eröffnen sich aus einer mediendidaktischen Perspektive. Es ist interessant zu fragen, welche Vorstellungen von Medien und Methoden für das Lehren und Lernen in den studentischen Ad-hoc-Metaphern zum Ausdruck gebracht werden. So werden Medien und Methoden in zahlreichen didaktischen Modellen als relevante Strukturmomente des Unterrichts markiert, die es u. a. bei der Planung und Analyse von didaktischem Handeln zu berücksichtigen gilt (z. B. Schulz 2006; Klafki 1997; Heinmann 1962). Die Frage dabei lautet, inwiefern diese didaktische Perspektive von den Studierenden bereits mitgedacht und in ihren Adhoc-Metaphern zum Lehren und Lernen zum Ausdruck gebracht wird. 
Eine entsprechend fokussierte Betrachtung der metaphorischen Konzepte sowie der einzelnen Ad-hoc-Metaphern macht dabei sichtbar, dass die Begriffe Methode und Medien zur Erläuterung ausgewählter Bedeutungsübertragungen nicht verwendet werden. Zudem finden sich in den exemplarischen Narrationen kaum metaphorische Elemente, welche auf die Bedeutung von Medien im Kontext von Lehren und Lernen in der Schule schliessen lassen. Methoden werden in den Ad-hoc-Metaphern implizit skizziert aber nicht weiter differenziert. Das Phänomen der Nichtthematisierung von Medien und Methoden kann auf verschiedenen Ebenen ein Diskussionsund Reflexionsanlass eröffnen. Mit dem Fokus auf Medien lassen sich u. a. die folgenden Fragen stellen:

- Welche Bedeutung spielen Medien für das eigene Lernen im Studium bzw. wie wurden und werden Medien und didaktische Methoden im Unterricht sowie in der Hochschullehre erlebt?

- Inwiefern verhindern/ermöglichen die gewählten Bildbereiche die Thematisierungvon Medien (und Methoden)? Inwiefern sind die identifizierten metaphorischen Konzepte erweiterbar oder alternative Metaphern möglich, um die didaktische Perspektive auf Methoden und Medien zum Ausdruck zu bringen?

- Welche Vorstellungen und Überzeugungen haben Lehramtsstudierende hinsichtlich der Verwendung von Medien und Methoden im Unterricht?

Die erste Fragestellung zielt auf die Selbstreflexion bzw. dient als Anlass zur Aufarbeitung der eigenen Erfahrungen. Der zweite Fragenkomplex widmet sich den Möglichkeiten und Grenzen von Ad-hoc-Metaphern als Artikulationsformat. So kann für die verschiedenen Vergleiche diskutiert werden, inwiefern die verwendeten Bildbereiche Möglichkeiten zur Thematisierung von Medien eröffnen bzw. welche alternativen Sprachfiguren denkbar sind. Ferner lässt sich fragen, welche Bedeutung Studierende Medien in Lehr-Lernkontexten zuschreiben und welche Metaphern sie für diese Vorstellungen verwenden.

\subsubsection{Gendergerechte Sprache der Metaphern}

Ein weiterer «analytischer Blick» kann auf die (kaum) vorhandene geschlechtergerechte Sprache in den Metaphern der Studierenden gerichtet werden. So lässt sich fragen, inwiefern die Gleichberechtigung von allen Geschlechtern in Schrift und Wort im Rahmen der Ad-hoc-Metaphern zum Ausdruck gebracht wird. Die Relevanz eines entsprechenden Sprachbewusstseins wird in zunehmend sichtbaren Handlungsempfehlungen und an Universitäten und Hochschulen betont (z. B. Universität zu Köln 2017; TU Darmstadt 2017). Zudem wird die Bedeutung einer geschlechtergerechten 
Sprache in der Schule auf Basis empirischer Studien gestützt (Vervecken et al. 2013; Vervecken und Hannover 2015)249.

Bei der Untersuchung der verschiedenen Ad-hoc-Metaphern unter der skizzierten Fragestellung wird $u$. a. sichtbar, dass die Darstellung von Lehrerinnen und Lehrern sowohl im Bildbereich als auch im Zielbereich unabhängig vom metaphorischen Muster überwiegend in männlicher Form erfolgte. Beispiele dafür finden sich in nahezu alle verwendeten Bildbereichen: «Reiseführer: Lehrer (Lehrt nach dem Plan)» (D15-19), «Der Lehrende (Bäcker)» (D15-18), «Lehrer als Gärtner» (D15-18) oder «Verkäufer ist der Lehrende» (D15-20). Hingegen wurden in keiner Ad-hoc-Metapher eine ausschliesslich weibliche oder geschlechtergerechte Schreibweise verwendet. Vor diesem Hintergrund eröffnen sich verschiedene Diskussions- und Reflexionsanlässe für Studierende:

- Inwiefern sind die Metaphern der Studierenden geschlechtergerecht formuliert?

- Inwiefern erscheint die Beschränkung auf (fast nur) maskuline Formen als hinreichender Beitrag zur Gleichberechtigung von allen Geschlechtern in Schrift und Wort?

- Inwiefern verhindern die gewählten Bildbereiche die Thematisierung von Frauen und Männern oder inwiefern reproduzieren die gewählten Bildbereiche Stereotype?

Die erste Fragestellung dient zur Sensibilisierung der verwendeten Ausdrucksweisen zur sprachlichen Darstellung der gewählten Metaphern. Die zweite Fragestellung dient der Schaffung eines Problembewusstseins bzw. als Anregung zur Selbstreflexion. Mit der Diskussion der dritten Fragestellung können weitere Anlässe zur Schaffung eines Problembewusstseins sowie zur Reflexion des eigenen Sprachgebrauchs angeregt werden.

\subsubsection{Möglichkeiten und Grenzen metaphorischer Vergleiche}

Explizite Vergleiche eröffnen die Möglichkeit, einen abstrakten Sachverhalt in einfachen - der unmittelbaren Erfahrung zugänglichen - Begriffen zu verstehen und dieses Verständnis zum Ausdruck zu bringen (Kap. 5.3.3, 5.5.3). Zugleich werden mit entsprechenden Metaphern nur bestimmte Aspekte des Sachverhaltes hervorgehoben, während andere Aspekte ausgeblendet werden. Diese grundsätzliche Eigenschaft von Metaphern und expliziten Vergleichen (Kap. 5.3.3) eröffnet verschiedene Diskussions- und Reflexionsanlässe zur Erweiterung der eigenen pädagogischen und didaktischen Artikulationsfähigkeit.

249 Beispielsweise zeigen Vervecken et al. (2013) sowie Vervecken und Hannover (2015) konkrete Effekte hinsichtlich der Wahrnehmung von Berufen durch Schülerinnen und Schüler der Primarstufe nach der Verwendung linguistischer Interventionen. Die Verwendung einer geschlechtergerechten Sprache im Rahmen der Interventionen eröffnet dabei Möglichkeiten, die Selbstwirksamkeitserwartungen von Kindern hinsichtlich traditionell männlich dominierter Berufe zu stärken. 
So kann für die jeweiligen metaphorischen Muster auf der Ebene des Zielbereichs zum einen gefragt werden, inwiefern nicht berücksichtige Elemente (z. B. Methoden und Medien) in den verschiedenen Bildbereichen übertragen werden können. Zum anderen kann diese Frage auch auf der Ebene des Bildbereichs erfolgen, d. h.: Welche nicht thematisierten Elemente des Bildbereichs lassen sich auf den Zielbereich des Lehrens und Lernens in der Schule übertragen ${ }^{250}$ ? Für das exemplarisch ausgewählte metaphorische Muster «Lehren und Lernen ist wie eine SICHTBARE FORTBEWEGUNG NACH PLAN» (Kap. 7.4.3.2) bieten sich u. a. folgende Fragen an:

- Bildbereich: Inwiefern wollten die Reisenden/Lernenden die Reise überhaupt unternehmen? Inwiefern konnten sie die Wahl des Reiseziels selbst treffen? Inwiefern ist eine Bedeutungsübertragung von Reiseunfällen und Reiseerkrankungen möglich und sinnvoll?

- Zielbereich: In welcher Weise wird eine Leistungsbewertung in den Ad-hoc-Metaphern berücksichtigt? Welche Rolle haben Methoden und Medien? Ist eine Bedeutungsübertragung für Konflikte im Unterricht und in der Schule möglich?

In der Diskussion dieser Fragen kann erkennbar werden, für welche Aspekte spezifische Ad-hoc-Metaphern und übergeordnete metaphorische Muster geeignet sind und für welche spezifischen Aspekte des Lehrens und Lernens in der Schule diese kaum übertragbar sind. In der expliziten Diskussion der Möglichkeiten und der Grenzen metaphorischer Vergleiche wird die Chance gesehen, Reflexionen über die eigenen Vorstellungen und deren Limitationen anzuregen. Um die Möglichkeiten und Grenzen metaphorischer Vergleiche aus didaktischer bzw. pädagogischer Perspektive angemessen einschätzen zu können, bedarf es zugleich Wissen über die jeweilige Perspektive. In dieser Hinsicht stellt Wissen über Didaktik und Pädagogik einerseits eine Voraussetzung dar. Anderseits kann die Auseinandersetzung mit diesen Fragestellungen ein Anlass sein, sich mit didaktischen und pädagogischem Wissen auseinanderzusetzen.

\subsubsection{Erarbeitung der Gruppenmetaphern}

Mithilfe verschiedener Aufgabenstellungen zur Diskussion und Zusammenarbeit in Kleingruppen wurde das Ziel verfolgt, dass sich Studierende mit alternativen Sprachfiguren und Perspektiven zum Lehren und Lernen auseinandersetzen (Kap. 6.4.1.2, Kap. 6.6.1.1). Mit der Anregung von Diskussionen wurde erwartet, einen Beitrag zur Reflexion und Bewusstwerdung der eigenen Vorstellungen zu leisten. Auf Basis der Analyse der Gruppenprodukte (Kap. 7.4.3.3, 7.4.3.4) und der Ad-hoc-Metaphern (Kap. 7.4.3.1, 7.4.3.2) lassen sich für die Auswertung und Interpretation folgende Aspekte hervorheben:

250 So lassen sich in Anlehnung an die Heuristiken von Schmidt und Taddicken (2017) vielfältige Fragestellungen generieren (Kap. 3.4.3). 
- Die Gruppenmetaphern basieren und erweitern die (metaphorischen Muster der) Ad-hoc-Metaphern hinsichtlich verschiedener Elemente der Bild- und Zielbereiche (Kap. 8.2.3.1).

- Verschiedene metaphorische (Teil-)Muster der Ad-hoc-Metaphern finden sich nicht in den Gruppenmetaphern, u. a. das metaphorische Muster der Materialbearbeitung (Kap. 8.2.3.2).

- Die Erläuterung der Gruppenmetaphern entspricht weitgehend den Darstellungsformen der Ad-hoc-Metaphern. Eine kritische Distanzierung erfolgt in Ansätzen (Kap. 8.2.3.3).

Die getroffenen Aussagen können als weiterführende Interpretationen der vorgelegten Analyseergebnisse (Kap. 7.4.3) und der Prozessreflexionen (Kap. 7.2) verstanden werden. Die verschiedenen Aspekte eröffnen interessante Einsichten zur Veränderung der formulierten Ad-hoc-Metaphern, geben Hinweise für mögliche Entwurfsmodifikationen und neue Forschungsfragen.

\subsubsection{Gruppenmetaphern als Erweiterung der Ad-hoc-Metaphern}

Beim Vergleich der Sprachfiguren und metaphorischen Muster der Gruppenmetaphern (Kap. 7.4.3.4) mit denen der Ad-hoc-Metaphern (Kap. 7.4.3.2) lassen sich vielfältige Anknüpfungspunkte markieren:

- Die Bildbereiche der Ad-hoc-Metaphern stellen den bildlichen Orientierungsrahmen für die Gruppenmetaphern dar.

- Die expliziten Vergleiche der Gruppenmetaphern wurden von den Ad-hoc-Metaphern (a) wörtlich übernommen, (b) leicht verändert oder (c) durch Ergänzungen präzisiert.

- Die Erläuterungen der Gruppenmetaphern orientieren sich häufig an den Ad-hocMetaphern, die hinsichtlich des wörtlichen Umfangs sowie der metaphorisch übertragenen Elemente ausdifferenziert wurden.

Die Gruppenmetaphern der Seminargruppe umfassen sechs verschiedene Bildbereiche bzw. Quellbereiche. Dazu gehören die Bildbereiche der Gartenarbeit (2), des Reisens (2), des Wanderns (1), einer Bäckerei (1), eines Hausbaus (1) sowie einer Radioübertragung (1). Diese Bildbereiche wurden bereits in den Ad-hoc-Metaphern verwendet, um die eigenen Vorstellungen über Lehren und Lernen zum Ausdruck bringen (Kap. 7.4.3.2, 8.2.2). Gruppenmetaphern mit Bezug auf weitere Bildbereichen finden sich nicht. In diesem Sinne basieren die Gruppenmetaphern auf den Bildbereichen der Ad-hoc-Metaphern.

Die Bezüge zwischen Ad-hoc-Metaphern und in Gruppen erstellten Metaphern werden über die Bildbereiche hinaus auch an konkreten Formulierungen der 
expliziten Vergleiche bzw. der Titel der Wikibeiträge für die Gruppenmetaphern erkennbar. Beispielsweise wurden konkrete Ad-hoc-Metaphern direkt übernommen. Dies zeigt sich exemplarisch an den Gruppenmetaphern Lehren und Lernen ist wie «Wandern» (G15-02) und "Senden und Empfangen» (G15-08), welche in identischer Formulierung bereits als Ad-hoc-Metapher verschriftlicht wurden. Weitere Gruppenmetaphern weisen sprachlich deutliche Ähnlichkeiten zu konkreten Ad-hoc-Metaphern auf. Die Ad-hoc-Metapher Lehren und Lernen ist wie «Häuser bauen ohne Bauplan» (D15-08) findet sich in erweiterter Fassung auch als Gruppenmetapher in der Formulierung Lehren und Lernen ist wie «Häuser bauen ohne konkreten Bauplan» (G15-04) wieder. Eine Erweiterung bzw. Ausdifferenzierung zeigt sich exemplarisch an der Gruppenmetapher mit dem Titel «Lehren und Lernen ist wie von einem Reiseführer begleitet werden» (G15-03). Zu Beginn des Textes wird darauf hingewiesen, dass die komplette Metapher wie folgt lautet: «Lehren und Lernen ist wie, wenn ein Reiseführer seine buntgemischte Reisegruppe auf holprigen Wegen durch den Nebel führt, bis alle wieder klar sehen können und die Wege wieder eben sind» (G1503). Eine Ad-hoc-Metapher lautete ebenfalls Lehren und Lernen ist wie «Wenn ein Reiseführer seine buntgemischte Reisegruppe auf holprigen Wegen durch den $\mathrm{Ne}-$ bel führt» (D15-20). Neben der Erweiterung von Ad-hoc-Metaphern finden sich auch Transformationen. Zur Gruppenmetapher Lehren und Lernen ist wie der «Besuch einer Bäckerei» (G15-07) findet sich die Ad-hoc-Metapher Lehren und Lernen ist «zum Bäcker gehen, was einzukaufen und zu essen» (D15-19).

Neben der Erweiterung der expliziten Vergleiche zeigt sich die Ausdifferenzierung der Ad-hoc-Metaphern insbesondere hinsichtlich des Umfangs der Erläuterungen sowie der metaphorisch übertragenen Elemente. Dies kann exemplarisch an der zuletzt benannten Ad-hoc-Metapher Lehren und Lernen ist wie «zum Bäcker gehen, was einzukaufen und zu essen» (D15-19) gezeigt werden. Die Erläuterung lautete:

«Der Verkäufer ist der Lehrende: Gibt Angebot/ Thema, kennt sich aus, macht schmackhaft; Käufer: Lernender: Wählt aus, entscheidet selbst, was von dem Angebot er an- und schließlich auch aufnimmt; Gebäck Angebot: Vor- (Zu)bereitete Themen und Inhalte (Zutaten); Bäckerei: Institution; Setzt ¿Überthemen> / Rahmen (Bäcker verkauft keine Autos)» (D15-19)

Im Rahmen der Gruppenmetapher wurden die verschiedene Elemente zum einen in Form szenischer Narrationen und expliziter Vergleiche ausführlicher beschrieben (G15-07). Zum anderen wurden weitere Aspekte übertragen, z. B. «Geld» zum Kaufen von Backwaren auf die zu erbringende «Anstrengung» beim Lernen. Weitere Aspekte sind u. a. der Hunger auf Backwaren als Motivation der Lernenden oder die Konkretisierung unterschiedlicher Backwaren: «Ein Brötchen beispielsweise steht für einen Lerninhalt, der relativ leicht zu erlernen ist. Ein Kuchen dagegen steht für einen Lerninhalt, der relativ schwer zu erlernen ist» (G15-07). Neben weiteren 
metaphorisierten Elementen ${ }^{251}$ wurden zudem die Grenzen verschiedener Metaphorisierungen thematisiert. Mit der Ergänzung weiterer Elemente zu den verschiedenen Ad-hoc-Metaphern gehen in Einzelfällen auch Bedeutungsverschiebungen und neue Akzentuierungen einher ${ }^{252}$.

\subsubsection{Gruppenprozesse als Filter für Sprachfiguren und metaphorische Muster?}

Neben der Erweiterung und Ausdifferenzierung der markierten Ad-hoc-Metaphern und metaphorischen Mustern ist auffällig, dass sich in den Gruppenmetaphern bestimmte metaphorische Muster und Sprachfiguren nicht identifizieren lassen. Beispielsweise finden sich in den Gruppenmetaphern keine Metaphorisierungen von Schülerinnen und Schülern als Rohmaterial, welche erst durch Lehrkräfte geformt werden können bzw. müssen. Obwohl das metaphorische Muster Lehren ist wie «MATERIALBEARBEITUNG UND OBJEKTBEWEGUNG» auf Basis verschiedener Adhoc-Metaphern rekonstruiert werden konnte (Kap. 7.4.3.2.2), werden keine Bezüge im Rahmen der Gruppenmetaphern dazu erkennbar. Weder die jeweiligen Bildbereiche noch das damit zum Ausdruck gebrachte Verständnis von Lehren wurde in den Gruppenprodukten thematisiert. Dieses Ergebnis ist deshalb interessant, da es kaum Erkenntnisse über die Transformation von metaphorischen Konzepten vorliegen. Wenngleich die vorliegenden Daten keine Aussage über die Transformationen der individuellen Vorstellungen erlauben, lässt sich markieren, dass die benannten Metaphern in den Diskussionen von den Studierenden nicht fortgeführt wurden.

\subsubsection{Möglichkeiten und Grenzen der gewählten Darstellungsformen}

Die Darstellungsformen zur Erläuterung der Gruppenmetaphern fielen in allen Gruppenmetaphern, bedingt durch die Aufgabenstellung, vielfältiger aus als die Darstellungen der Ad-hoc-Metaphern. Neben der Formulierung eines expliziten Vergleiches als Kurzfassung der Gruppenmetapher erfüllten die Studierenden die Aufgabe, eine tabellarische Übersicht mit den zentralen Elementen des Bildbereichs sowie ihren Entsprechungen des Zielbereichs zu formulieren. Weiterhin wurde die Metapher inklusive der markierten Elemente in Form eines Fliesstextes erläutert. Etwa bei der Hälfte der Gruppenmetaphern wurden die Grenzen der Metapher diskutiert (Kap. 7.4.3.3).

251 In diesem Fall wurde das Lernen in Gruppen sowie die Relevanz von Wissenschaftlerinnen und Wissenschaftlern im Zielbereich der Gruppenmetapher ergänzt.

252 Während Lehrende im metaphorischen Muster der Ad-hoc-Metaphern keinen unmittelbaren Zugriff auf die Lernenden als Pflanzen hatten, erhalten die Lehrenden in der Gruppenmetapher die Möglichkeit, Pflanzen zurechtzustutzen. Dies lässt den Schluss zu, dass mit der Gruppenmetapher auch andere Vorstellungen über die Handlungsmöglichkeiten von Lehrenden ausgedrückt werden. 
Bei konkreter Betrachtung des Fliesstextes zeigt sich, dass die Ausdrucksformen im Wesentlichen den Darstellungsformen der Ad-hoc-Metaphern entsprechen (Kap. 7.4.3.3). Die Studierenden wechselten zwischen szenischen Narrationen und Beschreibungen sowie expliziten Bedeutungsübertragungen ausgewählter Elemente. Ferner finden sich verschiedene Mischformen. Zudem zeigt sich erneut das Phänomen, dass routinisierte Metaphern zur Erklärung expliziter Metaphern verwendet wurden (Kap. 7.4.3.3). Ebenfalls lassen sich Hinweise dafür identifizieren, dass die Studierenden die Gruppenmetaphern arbeitsteilig erstellten und dadurch Brüche im Sprachstil zu erkennen sind.

Hinsichtlich der Aufgabenstellung, die Grenzen und Probleme der entwickelten Metaphern zu thematisieren, zeigt sich, dass das Aufgabenformat auch weitere Darstellungsformen erlaubt, die eine kritische Distanz zur eigenen Metaphorik zulässt. Zugleich wird sichtbar, dass nur etwa die Hälfte der Studierenden eine kritische Einschätzung der Grenzen ihrer entwickelten Metaphern im Rahmen der Wikibeiträge formulierten.

\subsubsection{Bildlichkeit der Gruppenmetaphern als potenzielle Reflexionsanlässe}

Im Rahmen des Kick-off-Events wurden die Studierenden aufgefordert, Videobotschaften zu erstellen, in denen sie die von ihnen entwickelten Gruppenmetaphern präsentieren (Kap. 6.6.1.1). Diese Videobotschaften waren Bestandteil einer optionalen Aufgabenstellung für Studierende der «Escola Superior de Educação de Viseu», welche im Studiengang «Artes Plásticas e Multimédia» eine Lehrveranstaltung von Nelson Gonçalves besuchten. Wenngleich im Rahmen der Veranstaltungen keine Interaktionen zwischen den Studierenden realisiert werden konnten, eröffneten die Reflexionen und Nachbereitung der Erfahrungen mit Nelson Gonçalves eine interessante mediengestalterische Perspektive auf die formulierten Metaphern. Bei der Betrachtung und Hinterfragung der expliziten Bildlichkeit der studentischen Gruppenmetaphern wird ein Reflexionspotenzial vermutet, um die eigenen Selbstverständlichkeiten zu hinterfragen bzw. zumindest zu irritieren. Zur Visualisierung und Animation der formulierten Metaphern stellten die Studierenden von Nelson Gonçalves vielfältige Fragen. Am Beispiel der Gartenmetaphorik, welche für die Videobotschaft auf Englisch mit «gardening» übersetzt wurde, hinterfragten die Studierenden z. B. folgende Aspekte:

«Ist es relevant ob der Gärtner ein Mann oder eine Frau ist? Ist es relevant ob es junge Gärtnerin ist oder ob es ein alter Gärtner ist? Ist die Vorstellung von einem Gärtner sowas wie eine industrielle Blumenproduktion, wie sie in den Niederlanden z. B. passiert? Ist das auch eine Vorstellung von (gardening)?» (Sprachmemo 019, 1:06-1:40 min, 14.07.2015). 
Fragestellungen wie diese eröffnen neue Handlungsspielräume, um Studierende zur Bewusstwerdung und Artikulation der eigenen Vorstellungen anzuregen. Zur Visualisierung und Animation der formulierten Metaphern müssen einerseits Gestaltungsentscheidungen getroffen werden. Anderseits sind mit diesen Entscheidungen auch Implikationen auf der Bedeutungsebene der Metaphern verbunden. Wenn Schülerinnen und Schüler als wachsende Pflanzen metaphorisiert werden, eröffnen sich unterschiedliche Möglichkeiten zur Visualisierung mit jeweils unterschiedlichen Implikationen. Beispielsweise kann ein Tulpenfeld, wie es in der Blumenproduktion in den Niederlanden zu sehen ist, deutlich andere Deutungen und Interpretationen hervorrufen als die Veranschaulichung von Pflanzen im Rahmen eines Schrebergartens. In der Konfrontation der Studierenden mit den entsprechenden Fragen wird die Möglichkeit gesehen, dass die eigenen Ad-hoc-Metaphern bzw. Gruppenmetaphern zum einen überdacht und präzisiert werden. Zum anderen besteht die Möglichkeit, dass die Grenzen der jeweils gewählten Metaphorik «sichtbar» werden.

\subsection{Mitgestaltung eines Wikibooks als Erfahrungs- und Reflexionsmöglichkeit}

Die (Weiter-)Entwicklung der Fähigkeit zur Teilhabe an einer partizipativen Medienkultur wurde für die Studierenden als eine Zielstellung im Rahmen des Entwurfs formuliert (Kap. 6.2.2). Im Sinne des Ansatzes einer integrativen Medienbildung wurde ein Blended-Learning-Konzept entwickelt, um Möglichkeiten zum Lernen mit und über soziale Medien im Allgemeinen sowie öffentlichen Wikis im Besonderen zu eröffnen. Die Gestaltung des Konzeptes orientierte sich an den begründeten Prinzipien zum Entwurf von Lehrhandlungen bzw. zur Gestaltung von Gelegenheitsstrukturen: (1.) Ermöglichung von Partizipation und neuen Erfahrungen (Kap. 6.5.3), (2.) Projektund Problemorientierung (Kap. 6.5.1) sowie (3.) Förderung von Kooperation und Kollaboration (Kap. 6.5.2).

Die Lehrhandlungen sowie konkreten Aufgabenstellungen wurden mit der Absicht entwickelt, dass die Studierenden neue Erfahrungen zum Lernen mit und über Medien machen können. Auf Basis dieser Erfahrungen kann eine Weiterentwicklung der eigenen Medienkompetenz im Sinne der Fähigkeit zur Teilhabe an einer partizipativen Medienkultur erfolgen (Kap. 6.2.2). Die erhobenen Daten zur Erfassung der Perspektive der Studierenden ermöglichen zwar keine unmittelbaren Rückschlüsse auf die Entwicklung der formulierten Kompetenzen (Kap. 6.4.2). Dafür bieten sie einen Einblick, wie die Studierenden die geschaffenen Lehr- und Lernsituationen wahrnahmen, wie sie damit umgingen und wie sie diese bewerteten. Auf Basis der dargestellten Ergebnisse der Interviews (Kap. 7.4.1), der Veranstaltungsevaluation (Kap. 7.4.2), der reflektieren Praxiserfahrungen (Kap. 7.2) sowie der Analyse der Versionsgeschichte des Wikis (Kap. 7.4.4) wird im Folgenden argumentiert, dass ... 
- die Durchführung eines Projektseminars zur Mitgestaltung eines öffentliches Wikibooks in Form des entwickelten Konzeptes vielfältige neue Erfahrungen für Studierende ermöglicht (Kap. 8.3.1),

- die kooperative und kollaborative Zusammenarbeit zur Formulierung gemeinsamer Texte für Studierende eine Herausforderung darstellt, welche sowohl als anregend und produktiv, aber auch als frustrierend erlebt werden kann (Kap. 8.3.2),

- die Gestaltung und Veröffentlichung eigener Wikibookbeiträge produktive Erfahrungen sowie ein Kompetenzerleben ermöglichen kann, sofern sich Studierende darauf einlassen (Kap. 8.3.3),

- die Mitgestaltung an einem öffentlichen Wikibook verschiedene Anlässe eröffnet, die Bedingungen der Wissensproduktion und -verbreitung in öffentlichen Wikis zu diskutieren und zu reflektieren (Kap. 8.3.4),

- die Nutzung und das Verständnis eines Wikibooks als Open Educational Resources (OER) neue Handlungsspielräume eröffnen kann (Kap. 8.3.5).

\subsection{1 «Kein 0815-Seminar»: Projektarbeit mit einem Wikibook als neue Erfahrung}

Die Entwicklung des Blended-Learning-Konzeptes (Kap. 6.6) orientierte sich an dem begründeten Prinzip der Erfahrungsorientierung sowie der Ermöglichung von Partizipation auf unterschiedlichen Ebenen (Kap. 6.5.3). Mit dem entwickelten Entwurf wurde in diesem Sinne zum einen das Ziel verfolgt, dass Studierende neue Erfahrungen zur Gestaltung institutioneller Lehre mit digitalen Medien erfahren können (Kap. 6.3.2). Zum anderen wurde das Ziel verfolgt, an Erfahrungen der Studierenden zum überwiegend «konsumierenden» Umgang mit Wikis anzuknüpfen und durch die handlungsorientierte Auseinandersetzung neue Erfahrungen zur Mitgestaltung von Wikis zu ermöglichen. Diese Erfahrungen können u. a. einen Anlass zur (Weiter-)Entwicklung der formulierten Kompetenzen (Kap. 6.4.2) eröffnen sowie die Reflexion der skeptischen Einstellungen gegenüber digitalen Medien unterstützen (Kap. 6.3.2). Auf Basis der Erprobung des Entwurfs sowie der Analyse der zwei durchgeführten Lehrveranstaltungen kann gezeigt werden, dass eine Vielzahl der Studierenden neue Erfahrungen zur Gestaltung institutioneller Lehre mit digitalen Medien sowie zum gestalterischen Umgang mit Wikis machen konnte.

Die Ergebnisse der Interviewanalyse zu den Einstellungen gegenüber der Projektidee (Kap. 7.4.1.1) sowie die Diskussion des Themas «Originalität und Innovation des Seminarkonzeptes» in den Gruppengesprächen (Kap. 7.3.2.2) illustrieren verschiedenen Erfahrungen, die Studierende im Rahmen der Seminare machen konnten. Im Vergleich zu anderen Seminaren wurde das Veranstaltungskonzept von Studierenden als Abwechslung bzw. neue Erfahrung beschrieben. Dabei verwiesen die Aussagen der Studierenden auf die Durchführung des Seminars als Projekt sowie der Produktorientierung im Sinne der gemeinsamen Formulierung und Veröffentlichung 
von Texten. Auch die Wahl der verwendeten Medien zur Durchführung der Seminare wurde von den Studierenden thematisiert.

Weiterhin zeigt sich, dass entsprechend der getroffenen Annahmen (Kap. 6.3.2) die Mehrheit der Studierenden zwar mit Wikis als Informationsquelle vertraut waren und beispielsweise Wikipedia verwendeten, sich jedoch nur einzelne Studierende aktiv an der Mitgestaltung von Wikis beteiligten. Dies belegen die Ergebnisse der Interviewanalyse zu den Vorkenntnissen und Vorerfahrungen der Studierenden (Kap. 7.4.1.2), die Diskussion des Themas «Wikibooks als neuer Erfahrungsraum vs. «Hochladeplattform » in den Gruppengesprächen (Kap. 7.4.2.2.2) sowie die Ergebnisse zur «Bewertung der Erfahrungen zur Mitgestaltung eines Wikibooks» der quantitativen Evaluation (Kap. 7.4.2.1.3). Beispielsweise war für 80 \% der Befragten das Schreiben an frei und öffentlich zugänglichen Texten etwas Neues (Kap. 7.4.2.1.3). In den Interviews wurde die Nutzung des Wiki-Editors (Kap. 7.4.1.2) ebenfalls als neu und unbekannt beschrieben.

Interessant ist in diesem Zusammenhang, dass Studierende im Rahmen der Gruppengespräche mehrfach den Wunsch äusserten, mehr über die Verwendung digitaler Medien für den Einsatz im Unterricht zu erfahren (Kap. 7.4.2.2.3). Über die Zielstellung zur (Weiter-)Entwicklung der eigenen Medienkompetenz hinaus, bietet die Thematisierung mediendidaktischer Fragen und Themen im Kontext eines Wikibookprojektes Anknüpfungspunkte an die Interessen der Studierenden ${ }^{253}$.

\subsubsection{Kollaboratives Schreiben in öffentlichen Wikis als Herausforderung}

In der Gestaltung kooperativer und kollaborativer Lernsituationen wurde im Entwurf die Möglichkeit gesehen, die zwei formulierten Zielstellungen zur Entwicklung einer pädagogischen Artikulations- und Reflexionsfähigkeit sowie die Fähigkeit zur Teilhabe an einer partizipativen Kultur verbinden zu können (Kap. 6.5.2). Mit der Entwicklung passender Aufgabenstellungen und Unterstützungsmöglichkeiten wurde das Ziel verfolgt, den Austausch der Studierenden über ihre eigenen Vorstellungen zu fördern (Kap. 6.4.1.2) sowie zur Diskussion pädagogischer Perspektiven beizutragen (Kap. 6.4.1.3). Ferner wurde das Ziel verfolgt, dass Studierende Erfahrungen zum gemeinsamen Lernen und Arbeiten mit digitalen Medien machen können und so Anlässe zur Reflexion des eigenen Medienhandelns eröffnet werden (Kap. 6.4.2.3). Auf Basis der Erprobung, des Entwurfs sowie der Analyse der zwei durchgeführten Lehrveranstaltungen kann gezeigt werden, dass die gestalteten Lernsituationen zwar einerseits entsprechende Erfahrungen ermöglichten. Anderseits wurden diese Erfahrungsmöglichkeiten nur von wenigen Studierenden wahrgenommen. Das

253 Im Rahmen des Seminars «Bildung metaphorisch verstehen» waren diese Rückmeldung vor allem deshalb interessant, weil die Auseinandersetzung mit digitalen Medien kein Bestandteil der zugehörigen Modulbeschreibung darstellt. 
gemeinsame Schreiben und Veröffentlichen von Texten mit digitalen Medien war für viele Studierende eine Herausforderung. Dies wird durch die beschriebenen Handlungsstrategien der Studierenden, durch ihre Bewertung der unterschiedlichen Gruppenaktivitäten sowie ihrer Wahrnehmung der Aufgabenstellungen und Unterstützungsmassnahmen sichtbar.

Die Ergebnisse der Interviewanalyse (Kap. 7.4.1.3), der Wikibookanalyse (Kap. 7.4.4) sowie die Praxisreflexionen zur Verwendung von Moodle durch die Studierenden (Kap. 7.2.2.2, 7.2.3.2, 7.2.4.2) zeigen, dass die Studierenden unterschiedliche Strategien zum Zusammenarbeiten wählten. Diese Strategien umfassten kollaborative und kooperativen Vorgehensweisen sowie arbeitsteilige Bearbeitungsstrategien (Kap. 7.4.1.3.1). Die Verwendung von digitalen Medien zur Gruppenorganisation sowie zum Austausch wurde von der Mehrheit der Studierenden für die zweite Seminarphase beschrieben. Die Gründung einer WhatsApp-Gruppe und/oder der Austausch über Moodle gehörte für die Mehrheit der Studierenden zum Vorgehen. Bei Betrachtung der Statistiken der Moodle-Foren (Kap. 7.2.2.2, 7.2.3.2) wird deutlich, dass sich einzelne Arbeitsgruppen über Moodle Dokumente und Texte austauschten und verbesserten. Weitere Studierende beschrieben in den Interviews das Versenden von Textdokumenten mit E-Mails sowie den Austausch von Dateien über Clouddienste (Kap. 7.4.1.3). Wenngleich Studierende den Wiki-Editor verwendeten, um ihre arbeitsteilig erstellten Ergebnisse zu veröffentlichen, wählte keine der studentischen Arbeitsgruppen die Strategie, die Texte kollaborativ im Wiki zu schreiben (Kap. 7.4.4). In der Reflexion der eigenen Handlungsstrategien formulierte gleichwohl eine Studierende, dass es zurückblickend sinnvoller gewesen wäre, die Möglichkeiten zur kollaborativen Texterstellung des Wiki-Editors zu verwenden, statt regelmässig Textdokumente zu versenden (Kap. 7.4.1.3.3). Diese Reflexion der eigenen Handlungsstrategien ist ein Beispiel dafür, dass die gewonnenen Erfahrungen zur Reflexion des eigenen Medienhandelns verwendet werden können und so zur Weiterentwicklung der Fähigkeit zur begründeten Auswahl und Nutzung von sozialen Medien zur Kooperation sowie zum Lernen beitragen (Kap. 6.4.2.3). Gleichwohl ist die entsprechende Reflexion auch ein Einzelfall im Interviewsample. Ein Grund dafür, dass diese Reflexion nur von wenigen Studierenden erfolgt, wird in der Anlage des Projektes vermutet. Es ist möglich, dass durch die Seminarkonzeption und die Gestaltung der Präsenzveranstaltungen bei den Studierenden der Eindruck entstand, dass das Wikibook vor allem als Plattform zur Veröffentlichung unseres Buchprojektes dienen sollte. Diese Problematik wird in den folgenden Kapiteln näher erläutert (Kap. 8.3.5, 9.2.3).

Die im Rahmen der Zusammenarbeit gewonnenen Erfahrungen, das Erleben des Schreibens von Texten in Gruppen sowie die gemeinsame Erstellung eines Wikibooks wurde von den Studierenden unterschiedlich bewertet. Dabei variieren die Einstellungen der Studierenden gegenüber Gruppenarbeitsphasen (Kap. 7.4.1.1). Ferner wurden die gestalteten Lernsituationen der Gruppenarbeitsphasen unterschiedlich 
bewertet. Während Studierende einerseits die Freiräume zur Selbst- und Gruppenorganisation als angenehm bewerteten, kritisierten andere Studierende die seltenen Präsenztermine und äusserten den Wunsch nach mehr Orientierungshilfen. Das Diskussionsthema «Freiräume vs. Strukturierungswünsche zur Gruppenarbeit» der Gruppengespräche illustriert die unterschiedlichen Bewertungen und Argumente der Studierenden (Kap. 7.4.2.2.6).

Als relevantes Problem wurde in den Gruppengesprächen die Passivität von Mitstudierenden diskutiert (Kap. 7.4.2.2.5). Dabei zeigen die Daten der quantitativen Evaluation Unterschiede zwischen der Zufriedenheit mit der Aktivität der Gruppenmitglieder sowie der didaktischen Massnahmen hinsichtlich der verschiedenen Seminarphasen (Kap. 7.4.2.1.2). Insbesondere in der zweiten Seminarphase äusserten sich die Studierenden im Mittel unzufriedener als in der ersten Phase, sowohl hinsichtlich der Aktivität ihrer eigenen Gruppenmitglieder als auch hinsichtlich der Gruppeneinteilung. Auf Basis dieser Rückmeldungen erfolgte eine Modifikation des Entwurfs (Kap. 9.2.2).

\subsubsection{Gestaltung eigener Produkte im Web}

Mit der gemeinsamen Gestaltung eines Wikibooks wurde das Ziel verfolgt, verschiedene Anlässe zur individuellen und kollaborativen Erstellung eigener Medienbeiträge in Form von Wikiseiten zu eröffnen. Diese handlungsorientierte Auseinandersetzung erfordert eine Artikulation der eigenen Vorstellungen sowie die Darstellung pädagogischer Modelle (Kap. 6.4.1.1, 6.4.1.3, 6.4.1.4). Zudem werden so Möglichkeiten zur Weiterentwicklung der Fähigkeit zur Teilhabe an einer partizipativen Kultur im Sinne des Kompetenzbereichs «Gestalten und Veröffentlichen eigener Wikibeiträge» eröffnet (Kap. 6.4.2.1). Auf Basis der Erprobung des Entwurfs sowie der Analyse der zwei durchgeführten Projektseminare wird sichtbar, dass sich in den ersten zwei Seminarphasen nur wenige Studierende mit der Erstellung und Bearbeitung von Wikiseiten beschäftigten. Das Erstellen und Bearbeiten der Wikiseiten wurde in verschiedenen Arbeitsgruppen als «Hochladen» durch eine Person beschrieben, welche für das Wikibook bzw. die Technik zuständig war. Das gemeinsame Schreiben und Veröffentlichen von Texten mit einem Wiki-Editor erschien vielen Studierenden als Herausforderung, der sich jedoch nur wenige Studierende aktiv stellten. Zugleich zeigt sich in den Interviews mit den «Wikibeauftragten», dass die gestalterische Auseinandersetzung mit dem Wiki-Editor diesen Personen Erfolgserlebnisse und Lernanlässe ermöglichte. Entsprechend muss die im Prozess erfolgte Planungskorrektur (Kap. 7.2.3.2), auf die individuelle Veröffentlichung zu verzichten, problematisiert werden.

Die Ergebnisse der Interviews (Kap. 7.4.1.3.3), der Wikibookanalyse (Kap. 7.4.4) sowie der Praxisreflexionen (Kap. 7.2.2.3, 7.2.3.3, 7.2.4.3) zeigen, dass die Interaktionen mit dem Wikibook weitgehend auf das Kopieren und Speichern bereits 
vorgeschriebener Texte durch wenige Studierende beschränkt blieben. Auch die Ergebnisse der Evaluation zeigen, dass nicht alle Studierende Texte im Wikibook gestalteten und veröffentlichten (Kap. 7.4.2.1.4). Die Schwierigkeit der Bedienbarkeit des Wikis wurde in der quantitativen Befragung unterschiedlich eingeschätzt (Kap. 7.4.2.1.3). In den Interviews äusserten sich mehrere Lehramtsstudierende, dass sie keine Vorkenntnisse und Erfahrungen hinsichtlich der Nutzung eines Wiki-Editors hatten und dies als Herausforderung empfanden (Kap. 7.4.1.2). Diese Herausforderung wurde u. a. als Irritationsanlass, der zur Reflexion der eigenen Fähigkeiten führte, beschrieben. Zugleich beschrieben Studierende Bewältigungs- und Ausweichstrategien, um sich dieser Herausforderung nicht stellen zu müssen. Das Schreiben und Veröffentlichen der Wikibeiträge wurde von freiwilligen «Technik-» bzw. «Wikibeauftragten» in den jeweiligen Gruppen übernommen oder es entstand eine Situation der "Verantwortungsdiffusion», in der sich einzelne bis wenige Gruppenmitglieder zur Beschäftigung mit dem Wikibook bereit erklärten (Kap. 7.4.1.3.3).

Die produktive Auseinandersetzung mit dem Wiki-Editor zur Veröffentlichung der Wikibeiträge wurde von den aktiven Studierenden retrospektiv als hilfreiche Erfahrung bewertet (Kap. 7.4.1.3.3). Diese habe dabei geholfen, ihnen die "Angst» vor der Mitgestaltung einer Wikiseite zu nehmen. Die eigene Gestaltung einer Seite im Internet mithilfe eines Wikis wurde als Erfolg beschrieben. Diese Beschreibungen lassen sich als Hinweis dafür deuten, dass die produktive Arbeit mit dem Wiki-Editor Anlässe für Lernhandlungen bieten, welche zur Weiterentwicklung der eigenen Medienkompetenz beigetragen können (Kap. 6.4.2.1) Diese Möglichkeiten nahmen im Rahmen des Seminars, wie bereits markiert, jedoch nur wenige Studierende wahr. So überrascht das Ergebnis der quantitativen Evaluation nicht, dass die Mehrheit der Studierenden (eher) nicht der Aussage zustimmte, dass das Mitschreiben an einem Wikibook ihnen mehr Sicherheit gab, sich zukünftig aktiv an Wikis zu beteiligen (Kap. 7.4.2.1.4).

Vor diesem Hintergrund muss die im Prozess getroffene Gestaltungsentscheidung, auf die Aufgabenstellung zur individuellen Mitgestaltung von Wikiseiten zu verzichten, als Problem markiert werden. Statt weitere Studierende durch eine obligatorische Aufgabe mit der aktiven Mitgestaltung einer Wikiseite zu konfrontieren, wurde den Studierenden die individuelle Mitgestaltung als optionale Aufgabe gestellt. Dabei zeigte sich, dass die Aufgabe weitgehend nur von Studierenden wahrgenommen wurde, die sich bereits als Wiki- und Technikbeauftragte an der Gestaltung des Wikibooks beteiligt hatten. Ein zentraler Grund für die im Prozesse vorgenommene Planungskorrektur war die Qualität der Zwischenergebnisse der Studierenden (Kap. 7.2.3.2).

Die durch das Wikibookprojekt ermöglichten produktiven und gestalterischen Erfahrungen sind ein Argument zur Fortführung des produkt- und projektorientierten Ansatzes. Aufgrund der markierten Probleme sowie der heterogenen Einschätzungen 
im Rahmen der quantitativen und qualitativen Evaluation (Kap. 7.4.2.1.3, 7.4.2.2.2) wurde die Einbindung des Wikibooks jedoch hinterfragt (Kap. 8.3.5) und eine Entwurfsmodifikation vorgenommen (Kap. 9.2.3).

\subsubsection{Einblicke in eine partizipative Sharing-Gemeinschaft}

Mit der Wahl von Wikibooks als öffentliche Sharing-Gemeinschaft (Kap. 4.3.3.3) und als Schwesterprojekt der Wikipedia wurde das Ziel verfolgt, Studierenden einen Einblick in die Bedingungen der Wissensproduktion und Verbreitung in sozialen Medien zu eröffnen (Kap. 6.4.2.2). So wurde erwartet, dass der Beginn eines neuen Buchprojektes von der Wikibook- Gemeinschaft wahrgenommen und kommentiert wird. Diese Form der Beteiligung sollte u. a. einen Anlass bieten, um mit Menschen ausserhalb des Seminars in Kontakt zu kommen, Charakteristika von Wikis erfahrbar werden zu lassen sowie technische, rechtliche und gesellschaftliche Bedingungen der Wissensproduktion in einer handlungsorientierten Vorgehensweise zum Thema zu machen (Kap. 6.4.2.4, 6.4.2.1 6.4.2.2). Auf Basis der Erprobung des Entwurfs sowie der Analyse der zwei durchgeführten Projektseminare kann gezeigt werden, dass die gewünschten Interaktionen mit Wikibookianerinnen und Wikibookianern in unterschiedlicher Weise stattfanden und so für Studierende erfahrbar und thematisierbar wurden. Insbesondere die Kommentare auf der Diskussionsseite des Wikibooks sowie die Überarbeitungen boten einen Anlass zur Diskussion der Kollaborationserfahrungen mit Menschen ausserhalb des Seminars. Diese Erfahrungen wurden von der Mehrheit der Studierenden gemacht und waren Bestandteil von Diskussionen und Reflexionen im Rahmen der Interviews sowie in den abschliessenden Gruppengesprächen.

Die Ergebnisse der Wikibookanalyse (Kap. 7.4.4) illustrieren, dass Interaktionen mit Wikibookianerinnen und Wikibookianern sowohl auf der allgemeinen Startseite und der Diskussionsseite des Wikibooks erfolgten als auch in Form von Korrekturen und Überarbeitungen der Gruppenseiten der Studierenden. Damit waren potenzielle Anlässe vorhanden, über technische Bedingungen und Prinzipien von Wikis zu sprechen. Diese bezogen sich zum einen auf die Konkrete Nutzung des Wiki-Editors sowie auf den Umgang mit der entsprechenden Syntax. Zum anderen konnten die von Iske und Marotzki (2010) allgemein formulierten Prinzipien «Reflexivität», «Prozessualität» und «Partizipation» im Rahmen der Veranstaltung an konkreten Beispielen zum Thema gemacht werden (Kap. 7.2.2.3, 7.2.3.3). In diesem Sinne hat das Wikibookprojekt zumindest potenziell Erfahrungs- und Reflexionsanlässe eröffnet, um Lernhandlungen zum «Durchschauen und Beurteilen von Bedingungen der Wissensproduktion und Verbreitung in öffentlichen Wikis» zu initiieren.

Die Analyse der Interviews (Kap. 7.4.1.4) sowie die Ergebnisse der Evaluation zeigen (Kap. 7.4.2.1.3, 7.4.2.2.2), dass die Mehrheit der Studierenden die Kommentare und Anmerkungen der Wikibookianerinnen und Wikibookianern wahrnahmen und 
sich damit auseinandergesetzt haben. Diese Erfahrungen waren der Ausgangspunkt für unterschiedliche Reflexionen der Studierenden, z. B. hinsichtlich der fachlichen Kompetenz der Mitglieder der Wikicommunity sowie bezüglich der Feedbackkultur. Die Bewertung der Interaktionen fiel unterschiedlich aus. Während in einer Gruppendiskussion die Interaktionen mit einem Mitglied der Wikicommunity problematisiert wurde (Kap. 7.4.2.2.2), erfolgten die Bewertungen der fachlichen Kompetenz sowie des Tonfalls in der quantitativen Evaluation eher neutral (Kap. 7.4.2.1.3). Wenngleich einzelne Studierende davon berichteten, dass sie die Kommentare der Wikicommunity nicht wahrnahmen, sensibilisierte die Interaktion mit Wikibookianerinnen und Wikibookianern mehrere Studierende dafür, dass ihre Texte öffentlich zugänglich sind und auch von Anderen gelesen werden (Kap. 7.4.1.4). Bei der aktuellen Erprobung erwies sich die Wahl von Wikibooks als geeignet, um einen Einblick in eine partizipative Medienkultur zu eröffnen.

\subsubsection{Mehr als ein Publikationsprojekt - neue Handlungsspielräume}

Auf Basis des erprobten Konzeptes sowie der analysierten Daten wurde gezeigt, in welcher Weise ausgewählte Ziele erreicht werden konnten, welche potenziellen Lernanlässe eröffnet und von Studierenden wahrgenommen wurden sowie welche praxisbezogenen Schwierigkeiten und Herausforderungen sich bei der Erprobung ergaben. Abschliessend wird argumentiert, dass die Gestaltung eines Wikibooks in der Logik eines klassischen Publikationsprojektes verschiedene Grenzen beinhaltet. Um neue Handlungsspielräume zu eröffnen, erscheint die Perspektive lohnenswert, die (Mit-)Gestaltung eines Wikibooks nicht als klassisches Publikationsprojekt zu verstehen sondern als Erstellung von freien und offenen Lehr- und Lernmaterialien zu betrachten.

Die Betrachtung des Wikibooks als freies und offenes Lehr- und Lernmaterial kann zunächst eine konzeptionelle Ebene zur Identifikation neuer Handlungsspielräume eröffnen. Im Rahmen der ersten Projektdurchführung wurde das Wikibook «from the scratch» bzw. von Anfang an entwickelt. Durch diese zu Beginn sehr offene Entwicklung konnten Studierende einerseits in verschiedene Gestaltungsfragen involviert werden, z. B. durch die Gestaltung eigener Wikiseiten ohne Formatvorlagen (Kap. 7.2.2) sowie durch Abstimmungen zum Layout und zur gendergerechten Sprache (Kap. 7.2.3). Anderseits wurde das Erstellen der Texte zwar von vielen Studierenden als neue Erfahrung beschrieben, zugleich aber auch als «Hochladeplattform» bzw. lediglich als Form der Ergebnispräsentation erlebt. Das gemeinsame Lesen von Textbeiträgen erfolgte im Rahmen der ersten Projektphase nur hinsichtlich der Kommentare durch die Wikibook-Community. Die Verwendung des Wikibooks als Lehrund Lernmaterial erfolgte nicht und stellt damit eine «Lücke» des aktuellen Entwurfs sowie der Erprobung dar. Da die Rahmentexte für die verschiedenen Kapitel ebenso 
wie die Texte der Studierenden im Prozess entstanden, konnten diese nicht zur Vorbereitung bzw. als Literaturhinweis auf die jeweiligen Seminarphasen verwendet werden. Mit dem aktuell vorliegenden Wikibook hat sich diese Situation geändert und bietet neue Erfahrungsmöglichkeiten für Studierende. Die vorliegenden Kapiteleinleitungen können als Einführungsliteratur für die Studierenden verwendet und zugleich hinsichtlich ihrer Eignung und Nachvollziehbarkeit durch die Studierenden kritisiert und gemeinsam verbessert werden. So eröffnen sich neue Handlungsspielräume, um Studierenden einen Einblick in die Prozessualität von Wikibeiträgen und Partizipationsmöglichkeiten von Wikis zu eröffnen (Kap. 9.2.3).

Eine zweite Ebene zur Identifikation neuer Handlungsspielräume widmet sich der instrumentellen Nutzung des Wikibooks im Rahmen der Präsenztermine sowie im Rahmen der selbst organisierten Schreibzeiten. Im Rahmen der ersten Projektdurchführung wurden den Studierenden viele Freiräume eröffnet. Mit diesen Freiräumen entstanden Probleme bei der Nutzung des Wiki-Editors, welche durch die Wikicommunity kommentiert und problematisiert wurden. Diese markierten Probleme konnten im Rahmen der Präsenztermine als Diskussionsanlässe zur Verwendung des Wikibooks (Kap. 6.4.2.1) sowie zu den Bedingungen der Wissensproduktion in öffentlichen Sharing-Community (Kap. 6.4.2.2) verwendet werden. Gleichwohl erfolgte die aktive Auseinandersetzung mit den Gestaltungsprinzipien des Wikibooks nur durch wenige Studierende. Statt der Eröffnung von Freiräumen und optionalen Tätigkeiten scheint es notwendig zu sein, Studierende mit einer Aufgabenstellung zur aktiven Mitgestaltung eines Wikis zu konfrontieren. Ferner erscheint es hilfreich, mehr Orientierungen und Hilfestellungen zur Verfügung zu stellen, um möglichst vielen Studierenden entsprechende Erfahrungen zu ermöglichen. Dies kann beispielsweise bei der gemeinsamen Erstellung eines Accounts - als ein Schritt zur Teilhabe an einer Community - beginnen und die Gestaltung und Überarbeitung der eigenen sowie anderer Wikiseiten umfassen (Kap. 9.2.3).

\subsection{Geltungsbegründung und Reflexion der Forschungsmethoden}

Die kritische Betrachtung der empirisch gewonnenen Forschungsergebnisse ist für Sesink und Reinmann (2015, 80 f.) ein weiterer zentraler Bestandteil dieser Forschungsphase. Diese Überlegungen sind anknüpfungsfähig an den im Kapitel Erwartungen der scientific community (Kap. 2.2.1) formulierten wissenschaftlichen Anspruch zum «Einhalten von Gütekriterien und Qualitätsstandards» bei der Durchführung gestaltungsorientierter Bildungsforschung, wie Tulodziecki et al. $(2013,230)$ markieren. Dafür formulieren Sesink und Reinmann $(2015,81)$ Orientierungsfragen auf methodischer wie methodologischer Ebene. Beispielsweise steht für die «angewandten empirischen Verfahren», so Sesink und Reinmann (2015, 81), «praktisch deren methodisch «saubere) Durchführung in Frage». Unter Berücksichtigung der 
verwendeten Forschungsmethoden widmet sich das folgende Kapitel einer kriteriengeleiteten Betrachtung der empirischen Ergebnisse mithilfe der Gütekriterien qualitativer Forschung (Steinke 2012; Flick 2012).

\subsubsection{Intersubjektive Nachvollziehbarkeit}

Für die verwendeten qualitativen Forschungsmethoden kann in Anlehnung an Steinke $(2012,324)$ der Anspruch auf intersubjektive Nachvollziehbarkeit erhoben werden. Auf dieser Basis kann, so Steinke (2012, 324), «die Bewertung der Ergebnisse erfolgen». Zur «Sicherung und Prüfung» unterscheidet Steinke (2012) drei verschiedene Herangehensweisen, welche im Folgenden jeweils diskutiert werden: «Dokumentation des Forschungsprozesses» (ebd., 324), «Interpretation in Gruppen» (ebd., 326) sowie «Anwendung kodifizierter Verfahren» (ebd., 326).

Zur Dokumentation des Forschungsprozesses erfolgte eine Beschreibung der verwendeten Erhebungsmethoden, der Strategien zur Datenaufbereitung (z. B. A.1.2), des Erhebungskontextes bezüglich der problemzentrierten Interviews (Kap. 3.3.2), des verwendeten Fragebogens und der Durchführung der Gruppengespräche als Veranstaltungsevaluation (Kap 3.3.3) sowie der studentischen Seminarprodukte (Kap. 3.3.4, 3.3.5). Spezifische Anpassungen der verwendeten Erhebungsmethode sowie eine Konkretisierung des Datenmaterials wurden im Rahmen der zugehörigen Fallstudie beschrieben (Kap. 7.3). Ferner sind die anonymisierten Transkripte der Interviews sowie der Gruppengespräche im Anhang dokumentiert (Anhang B.6, B.5 C.7, C.7). Ebenfalls im Anhang vorhanden sind die Daten zur Metaphernanalyse (Anhang B.1, C.1), zur quantitativen Evaluation (Anhang B.4, C.4) sowie zum Wikibook (Anhang E). Die Beschreibung des Vorgehens sowie die Dokumentation der Daten erfolgte mit dem Ziel, die dargestellten Ergebnisse in ihrem Entstehungszusammenhang nachvollziehen zu können.

Die «Interpretation in Gruppen» erfolgte zum einen in Zusammenarbeit mit studentischen Mitarbeiterinnen und Mitarbeitern ${ }^{254}$. Zum anderen erfolgte zur kommunikativen Validierung verschiedener Auswertungsschritte eine Diskussion der aufbereiteten Daten und Ergebnisse in zwei regelmässigen Forschungswerkstätten. Die erste Forschungswerkstatt «Medienpädagogik» wurde in Zusammenarbeit mit Petra Grell organisiert und richtete sich an Studierende und Promovierende im Bereich der

254 Die Einschätzung der studentischen Metaphern und Rekonstruktion erfolgte mit zwei studentischen Mitarbeiterinnen. Exemplarische Materialien zur Dokumentation der Treffen zur formalen Einschätzung der Gruppenmetaphern sind im Anhang dokumentiert (Anhang B.1). Die Auswertung der Interviews erfolgte im Austausch mit einem studentischen Mitarbeiter zur Entwicklung des Kodierleitfadens (Anhang A.1.3) sowie zur Diskussion kodierter Textstellen. Mit einer weiteren studentischen Mitarbeiterin erfolgte die systematische Aufbereitung der Gruppengespräche (Anhang B.5), es wurden verschiedene Auswertungsverfahren erprobt und deren jeweilige Eignung diskutiert. 
Allgemeinen Pädagogik mit dem Schwerpunkt Medienpädagogik ${ }^{255}$. Die zweite Forschungswerkstatt wurde von wissenschaftlichen Mitarbeiterinnen und Mitarbeitern des Instituts für Allgemeine Pädagogik und Berufspädagogik selbst organisiert ${ }^{256}$. Im Rahmen dieser Forschungswerkstätten wurden u. a. folgende Materialien und Auswertungsstrategien diskutiert: Strategien zur Rekonstruktion metaphorischer Muster der Ad-hoc-Metaphern und Gruppenmetaphern, Nachvollziehbarkeit des erstellten Leitfadens zur kategorienbasierten Auswertung der Interviews (Anhang A.1.3), Vorgehen zur Auswertung und Zusammenfassung der Gruppengespräche, Darstellungsformat der quantitativen Auswertung der Veranstaltungsevaluation sowie Strategien zur selektiven Plausibilisierung der Interviewergebnisse und Gruppendiskussion. Unter Berücksichtigung aller Auswertungsschritte und Forschungsphasen wurde in insgesamt 46 wissenschaftlichen Forschungswerkstätten und -kolloquien konkretes Datenmaterial besprochen und das jeweilige methodische Vorgehen diskutiert.

Die «Anwendung kodifizierter Verfahren» umfasst für Steinke $(2012,326)$ den Rückgriff auf regelgeleitete Forschungsmethoden. Wenngleich im Umgang mit qualitativen Daten kaum standardisierte Vorgehen existieren können, lassen sich strukturierte Vorgehensweisen identifizieren, welche zur intersubjektiven Nachvollziehbarkeit des forschungsmethodischen Vorgehens beitragen können. Im Kapitel Qualitative Inhaltsanalysen (Kap. 3.4.1) wurden dafür die Techniken der zusammenfassenden sowie der strukturierenden Inhaltsanalyse skizziert, welche für die Auswertung der Gruppengespräche sowie der Interviews verwendet wurden. Zur Rekonstruktion metaphorischer Muster wurde ein Verfahren adaptiert, welches im Kontext deutschsprachiger qualitativer Sozialforschung verhältnismässig unbekannt ist. Entsprechend wurden für dieses Vorgehen, wie von (Steinke 2012, 326) gefordert, die einzelnen Analyseschritte expliziert und im Detail dokumentiert.

\subsubsection{Angemessenheit des Forschungsprozesses}

Als zweites Gütekriterium benennt Steinke $(2012,326)$ die «Indikation des Forschungsprozesses». Darunter versteht Steinke (2012, 326 ff.) die Angemessenheit des gesamten Forschungsprozesses. Dieses Kriterium ist anschlussfähig an die im Kapitel "Charakteristika gestaltungsorientierter Bildungsforschung» (Kap. 2.2) formulierten Prozessstandards. Gleichwohl unterschiedet sich ein qualitativer Forschungsprozess, wie ihn Steinke $(2012,326)$ skizziert, hinsichtlich verschiedener Aspekte von einem entwicklungsorientierten Forschungsprozess (Kap. 3.1). Während die Berücksichtigung relevanter Prozessstandstandards für entwicklungs- und gestaltungsorientierte

255 Zwischen den Jahren 2015 und 2018 wurde die Veranstaltung mindestens alle zwei Wochen durchgeführt und über gemeinsame Forschungsprojekte, Abschlussarbeiten und konkrete Datenauswertungen diskutiert.

256 Die Forschungswerkstatt wurde zwischen den Jahren 2017 und 2019 mehrmals pro Semester durchgeführt. 
Projekte im Rahmen der methodischen Verortung der einzelnen Kapitel diskutiert werden, widmen sich die folgenden Ausführungen den von Steinke (2012, 326 ff.) benannten Aspekten zur «Indikation des qualitativen Vorgehens angesichts der Fragestellung», zur «Indikation der Methodenwahl», zur «Indikation der Samplingstrategien» sowie zur «Indikation der methodischen Einzelentscheidungen im Kontext der gesamten Untersuchung».

Die Angemessenheit überwiegend qualitativer Zugänge begründet sich für das vorliegende Forschungs- und Entwicklungsprojekt sowohl hinsichtlich der Fragestellung (Kap. 3.2) sowie hinsichtlich des gewählten methodologischen Ansatzes zur entwicklungsorientierten Bildungsforschung (Kap. 2.4). Mit verschiedenen Metaphern beschreiben Sesink und Reinmann (2015) das Ziel dieses Ansatzes wie folgt: «lhre Intention ist nicht abschließend, sondern aufschließend, nicht feststellend, sondern 〈bewegend»» (Sesink und Reinmann 2015, 81). Die Durchführung eines pädagogischen Experimentes sowie dessen systematische Untersuchung und theoretische Reflexion richtet sich für Sesink und Reinmann $(2015,82)$ «besonders auf jene Prozesserfahrungen, aus denen sich für die Weiterentwicklung der Praxis neue Perspektiven entwickeln lassen». Die Fokussierung von Prozesserfahrungen bzw. einer Prozessorientierung gegenüber «feststellender» Ergebnisorientierung beschreibt von Kardorff $(2012,245)$ als methodologisches Prinzip qualitativer Evaluationsforschung. In diesem Sinne empfehlen Sesink und Reinmann $(2015,82)$ die Verwendung offener Erhebungsverfahren:

«Methodisch werden daher «Evaluationsverfahren» benötigt, die einen solchen Verlauf nicht nur zulassen, sondern möglichst sogar unterstützen. Neben den Verfahren, die sich zur Bestätigung oder Nicht-Bestätigung von Hypothesen prognostischen Typs eignen, gehören hierzu auch solche Verfahren, welche eine sowohl individuelle als auch diskursive Reflexion des Prozessverlaufs anregen, sicher stellen (sic) und dokumentieren, so dass insbesondere nicht Vorher-Gedachtes, Unerwartetes wahrgenommen und einer Verständigung darüber zugeführt wird, welche Bedeutung ihm für das gemeinsame Projekt zugeschrieben wird. Forschungstagebücher, regelmäßige Teamsitzungen, deren TO Raum für das Einbringen neuer Erfahrungen bietet, Sitzungsprotokolle, welche Konsens und Differenzen der Teilnehmer/innen über den Prozessverlauf und die je zu aktualisierende Perspektivierung dokumentieren, könnten geeignete Instrumente sein» (Sesink und Reinmann 2015, 82).

Mit der Verwendung eigener Sprachmemos zur Dokumentation der Praxis- und Forschungserfahrungen (Kap. 3.3.1), der Durchführung von problemzentrierten bzw. episodischen Interviews im Verlauf des Seminars (Kap. 3.3.1) sowie einer qualitativen Veranstaltungsevaluation (Kap. 3.3.3.1) sowie der Analyse der Versionsgeschichte des Wikibooks (Kap. 3.4.2.2) wurde versucht, dem methodologischen Anspruch 
auf Prozessorientierung und Offenheit gerecht zu werden. So eröffneten die vier benannten Erhebungsmethoden sowie die damit gewonnenen Daten unterschiedliche Möglichkeiten Aussagen über den Prozessverlauf des Seminars zu treffen. Ferner eröffneten die Durchführung der Interviews und Gruppengespräche für die Teilnehmerinnen und Teilnehmer die Möglichkeit, ihre Erfahrungen und Interessen zum Wikibookprojekt zum Ausdruck zu bringen. Mit der Kombination der unterschiedlichen Verfahren wurde zudem der Anspruch verfolgt, dem von Tulodziecki et al. $(2013,229)$ formulierten Prozessstandard zur «Verknüpfung unterschiedlicher Vorgehensweisen bei der Datenerfassung und Auswertung» zu genügen (Kap. 2.2.1).

Die Angemessenheit bzw. die «Indikation der Samplingstrategien» umfasst zwei Fragen: «Inwiefern ist die Auswahl der Untersuchungsfälle, -situationen etc. indiziert?» und «lst das Sampling zweckgerichtet, werden informationsreiche Fälle ausgewählt?» (Steinke 2012, 328). Diese Fragen sind für die verschiedenen Erhebungsmethoden unterschiedlich zu beantworten. Die Analyse der Verlaufsgeschichte des Wikibooks umfasst alle erstellten Wikiseiten im Rahmen des Seminars und kann in diesem Sinne als Vollerhebung verstanden werden. Gleichwohl beteiligten sich nicht alle Studierenden an der Gestaltung des Wikibooks. Die Durchführung der Veranstaltungsevaluation sowie die Erhebung der Ad-hoc-Metaphern können als Gelegenheitsstichprobe im Rahmen des Seminars beschrieben werden (Kap. 7.3.2.2, 7.3.2.1). Die Teilnahme an den benannten Erhebungsmethoden erfolgte jeweils nur von anwesenden Studierenden. Ferner wurde zur Durchführung der quantitativen Evaluation der Versuch unternommen, über eine Online-Umfrage mehr Studierende zur Teilnahme zu erreichen. Dies gelang jedoch mit zwei Teilnehmerinnen und Teilnehmer an der Online-Umfrage nicht. Für die Auswahl von Interviewpartnerinnen und -partner wurde versucht, Personen aus unterschiedlichen Seminaren sowie jeweils aus Arbeitsgruppen zu erreichen. Da der Zugang sowie die Ansprache potenzieller Interviewteilnehmerinnen und -teilnehmer insbesondere über das Präsenzseminar erfolgte, bestand die gleiche Problematik wie bei der Durchführung der Evaluation. Es konnten nur die Studierenden angesprochen werden, die im Zeitraum der Interviewdurchführung auch in den Präsenzveranstaltungen anwesend waren (Kap. 7.3.1). Gleichwohl ist es mit der Durchführung von elf Interviews gelungen, jeweils Studierende aus unterschiedlichen Arbeitsgruppen zu interviewen. So konnte ein Einblick in die verschiedenen Vorgehensweisen der Gruppen gewonnen werden.

Die von Steinke $(2012,328)$ benannte «Indikation der methodischen Einzelentscheidungen im Kontext der gesamten Untersuchung» umfasst zwei Fragen: Die Passung zwischen Erhebungs- und Auswertungsverfahren sowie die Passung vom Untersuchungsdesign zu den verfügbaren Ressourcen. Während die Angemessenheit der einzelnen Verfahren zur Datenerhebung und -auswertung in den Kapiteln «Zugang zum «Feld» und Methoden der Datenerhebung» (Kap. 3.3) und «Methoden zur Analyse und Interpretation» (Kap. 3.4) diskutiert wurde, muss die Passung zwischen 
dem Untersuchungsdesign und den verfügbaren Ressourcen an dieser Stelle thematisiert werden. So wurde geplant, das Projektseminar in zwei aufeinander folgenden Semestern mit jeweils zwei thematischen Schwerpunkten durchzuführen. Trotz der Unterstützung des Projektes durch eine studentische Mitarbeiterin als Tutorin in der Praxis wurde der Arbeitsaufwand im Sommersemester 2015, der nötig war, um sowohl der Rolle des Lehrenden als auch der Rolle des Forschenden zu genügen, unterschätzt. Aufgrund des begrenzten Zeitraums zur Auswertung der Daten sowie zur Modifikation des Entwurfs entstand ein Handlungsdruck, beiden Rollen gerecht zu werden, der nur schwer zu bewältigen war. Dies dokumentiert das folgende Sprachmemo im September 2015:

"Was mich auch ein wenig belastet oder stört, ist sozusagen die (kurze Pause) einerseits weiterhin Beratungsaufwand sowie Korrekturaufwand zu haben (kurze Pause) oder es ist eher so Feedbackaufwand, also diese Reviews schreiben mit Kommentaren an den Texten sind ja schon weniger rein der Kontrolle und ( Ja, ist gut.) oder ‘ Nein, ist nicht gut.), sondern über diese Feedbackbögen auch eine Rückmeldung zu geben. Und das ist schon irgendwie belastend einerseits die Daten auszuwerten und andererseits (kurze Pause) genau noch Lehre parallel zu machen. Also das ist irgendwie in der Konzeption dieser Untersuchung (kurze Pause) also das ist echt schwierig unter einen Hut zu kriegen» (Sprachmemo 019d, 2:10-3:12 min, 04.09.2015).

Zum Umgang mit dieser Schwierigkeit mussten hinsichtlich der Auswertung und Interpretation der vielfältigen Daten verschiedene Auswahlentscheidungen getroffen werden. So erfolgte die Interpretation der Daten überwiegend - wie es Sesink und Reinmann $(2015,81)$ formulieren - «rückwärtsgewandt in Bezug auf die zuvor aufgestellten Hypothesen über den Zusammenhang von pädagogischem Handeln und darauf erfolgendem Prozessverlauf». Ferner wurde versucht zu skizzieren, inwiefern sich für die Lehrenden und Lernenden neue «Handlungsspielräume» eröffnet haben (Kap. 8.2.2, 8.2.4 8.3.5). Zugleich erfolgte dies jedoch hinsichtlich ausgewählter Schwerpunkte. Die Interviewauswertung wurde sich z. B. auf die studentische Perspektive zur Wahrnehmung und zum Umgang mit dem Wikibook beschränkt, wenngleich durch die Interviewdaten weitere Materialien zur Verfügung standen. Auf die Entwicklung neuer Ideen für die pädagogische Forschung, so lautet der Anspruch von (Sesink und Reinmann 2015, 81), wurde im Rahmen der ersten Iteration jedoch verzichtet. 


\subsubsection{Empirische Verankerung durch selektive Plausibilisierung}

In Anlehnung an Steinke $(2012,328)$ erfolgte die empirische Verankerung der dargestellten studentischen Perspektiven und Strategien im Umgang mit dem Wiki-Editor und der Wiki-Community (Kap. 7.4.1) zur Einschätzung des Seminars (Kap. 7.4.2.2) sowie zu Rekonstruktion metaphorischer Muster (Kap. 7.4.3) durch das Bereitstellen hinreichender Textbelege. Die Darstellung der Ergebnisse erfolgte möglichst «dicht an den Daten» (Steinke 2012, 328). Mit dieser Strategie zur selektiven Plausibilisierung, wie sie von Flick (2012, 488 ff.) bezeichnet wird, bedarf es auch einer Thematisierung des Anspruchs an Verallgemeinerung der Ergebnisse, welche im Folgenden unter den von Steinke (2012, 329 f.) markierten Gütekriterien «Limitation» und «Relevanz» diskutiert werden.

\subsubsection{Limitation, Kohärenz und Relevanz}

Die drei von Steinke $(2012,329$ f.) benannten Kriterien widmen sich insbesondere der Einschätzung entwickelter Theorien durch qualitative Forschungsmethoden. Mit dem Kriterium der «Limitation» stellt sich für Steinke (2012, 329 f.) die Frage nach dem Geltungsbereich der entwickelten Theorie. Das Kriterium «Kohärenz» dient zur Prüfung des Umgangs mit Widersprüchen bei der Theorieentwicklung und mit dem Kriterium der «Relevanz» fragt Steinke (2012, 329 f.) nach dem pragmatischen Nutzen einer entwickelten Theorie.

Die erste Durchführung und systematische Untersuchung des pädagogischen Experimentes erfolgte u. a. mit dem Ziel, Erkenntnisse «in Bezug auf die zuvor aufgestellten Hypothesen über den Zusammenhang von pädagogischem Handeln und darauf erfolgendem Prozessverlauf» (Sesink und Reinmann 2015, 81) zu gewinnen. Zudem konnten Aspekte identifiziert werden, welche neue Handlungsspielräume für die Gestaltung zukünftiger Praxis eröffnen und in einem modifizierten Entwurf münden (Kap. 9). Eine Auseinandersetzung mit der Frage, inwiefern die dargestellten Ergebnisse einen Beitrag zur Theorieentwicklung für die pädagogische Forschung leisten, stand im Rahmen der Fallstudie nicht im Fokus und wird zum Abschluss des Gesamtprojektes thematisiert (Kap. 11.6). Entsprechend wird an dieser Stelle darauf verzichtet, Aussagen über die Kohärenz und die Limitation ausführlich zu thematisieren. Mit dem Begriff «Fallstudie» kann jedoch in Anlehnung an Sesink und Reinmann $(2015,79)$ an dieser Stelle bereits darauf hingewiesen werden, dass der Geltungsbereich der gewonnenen Erkenntnisse als eingeschränkt betrachtet werden muss. So erfolgte die Durchführung der ersten Erprobung, systematischen Untersuchung sowie der Auswertung und Neuperspektivierung unter den spezifischen Rahmenbedingungen von Lehrveranstaltungen im Lehramtsstudium an der TU Darmstadt. 


\subsubsection{Reflektierte Subjektivität}

Als letztes Gütekriterium benennt Steinke (2012, 330 f.) die Reflexion der «konstituierenden Rolle des Forschers als Subjekt [...] und als Teil der sozialen Welt, die er erforscht [...]». Für die Durchführung des vorliegenden Projektes ist es ferner relevant zu berücksichtigen, dass sich die Reflexion der Subjektivität neben der Rolle des Forschenden auch auf die Rolle des Lehrenden bezieht (Kap. 2.4). Für Steinke (2012, 331) gilt es im ersten Schritt zu prüfen, inwiefern «der Forschungsprozess durch Selbstbeobachtung begleitet» wird. Dies erfolgte im Rahmen des Projektes durch die Aufzeichnung von Sprachmemos sowie das Führen von Forschungstagebüchern (Kap. 3.3.1). Im Kapitel «Erprobung des Entwurfs als Prozessreflexion» (Kap. 7.2) werden Auszüge aus den Memos verwendet, um die Perspektive als Lehrender zu thematisieren und zu reflektieren. Ferner beinhalten die Memos in Form von Sprachnotizen und Textbeiträgen verschiedene Überlegungen zur Datenauswertung in der Rolle als Forscher.

Das zweite Reflexionsmoment umfasst für Steinke $(2012,331)$ die Berücksichtigung der persönlichen Voraussetzungen zur Erforschung des Gegenstandes. Eine Auseinandersetzung mit den eigenen Vorannahmen und Fähigkeiten erfolgte zum einen durch die Nachbereitung der verschiedenen Erhebungsmethoden, z. B. in Form von Postskripten für die einzelnen Interviews. Durch die Dokumentation und Reflexion des eigenen Erlebens der Interviewsituation gelang es u. a. Probleme in der Interviewführung aufzudecken, z. B. hinsichtlich der Formulierung von Suggestivfragen. Zum anderen eröffnete die Teilnahme an den beschriebenen Forschungswerkstätten weitere Reflexionsanlässe. Exemplarisch wurde dies bei der gemeinsamen Diskussion des eigenen Datenmaterials sichtbar, z. B. hinsichtlich der studentischen Ad-hoc-Metaphern und Gruppenmetaphern. Der Argumentation von Gansen (2010) (Kap. 5.3.3) zur Unbestimmbarkeit von Metaphern entsprechend, zeigten sich in der Diskussion einzelner metaphorischer Redewendungen sehr unterschiedliche Lesarten und Interpretationsmöglichkeiten. Diese unterschiedlichen Lesarten lassen sich in Anlehnung an Gansen (2010) auf die unterschiedlichen Erfahrungen und Wissensbestände zurückführen. Was für einen Menschen als sehr konkret verstanden wird, kann für Menschen ohne Hintergrundwissen sehr abstrakt sein. Durch die kommunikative Validierung gelang es für viele metaphorische Ausdrücke, intersubjektiv nachvollziehbare Sprachfiguren und Muster zu rekonstruieren.

Das dritte und vierte Reflexionsmoment umfasst die «Vertrauensbeziehung zwischen Forschung und Informant» sowie den «Feldeinstieg» (Steinke 2012, 331). Im Kontext dieses entwicklungsorientierten Bildungsforschungsprojektes sind die Aspekte insbesondere deshalb relevant, weil im Forschungsfeld zwei verschiedene Rollen eingenommen wurden, die des Lehrenden und die des Forschenden. Die Erhebungssituation des Interviews war entsprechend eine durchaus spannungsvolle Situation (Kap. 3.3.2). Während die Studierenden sich einerseits freiwillig für die 
Teilnahme an einem Interview entschieden hatten, wurden sie anderseits von der Lehrperson interviewt, welche ihre Studienleistungen bewertet. Wie in der Konzeption der Interviewsituation beschrieben, erfolgte der Versuch, dieses Spannungsfeld zu bearbeiten, wenngleich es in gestaltungs- und entwicklungsorientierten Forschungsansätzen kaum auflösbar erscheint. In Betrachtung der sehr offenen und problematisierenden Aussagen der Studierenden zum Umgang mit dem Wikibook und dem Erleben des Seminars (Kap. 7.4.1) wird davon ausgegangen, dass die Interviewsituation zur Erhebung studentischer Perspektiven geeignet war. So kann die Formulierung einer interviewten Person: «[...] lass uns jetzt irgendwas schreiben, dass wir die Scheisse hinter uns haben» (B06, 61), in einer Interviewsituation mit dem Dozenten als exemplarischer Indikator dahingehend gedeutet werden, dass die Studierenden keine negativen Konsequenzen befürchteten.

Eine weitere Situation in der sich die eingenommene Doppelrolle als Lehrender und Forscher spannungsvoll gestaltete, war die Gestaltung von Seminarsitzungen. Insbesondere das Verhältnis von einem thematischen Abschluss in der letzten Präsenzveranstaltung zu einer angemessenen Erhebung von Daten (Kap. 3.3.3.1, 7.3.2.2) erwies sich im Verlauf des Projektes als schwierig. Aus der Perspektive des Lehrenden wurden die Planungsanpassungen wie folgt in einem Sprachmemo dokumentiert:

«[...] in welchem Verhältnis in meiner letzten Sitzung Evaluationsaspekte kommen sollten, könnten oder müssten oder eben eine inhaltliche Reflexion stattfinden soll. Weil als Lehrender habe ich irgendwie das Bedürfnis die Veranstaltung «rund» zu machen, d. h., noch mal aufzuzeigen, was der Sinn der verschiedenen Facetten war. Es kam ja in dem einen Interview mit [...] schon mal raus, dass irgendwie erst am Ende es jetzt wirklich Sinn gemacht hat, diese unterschiedlichen Phasen zu begehen. Wobei eben [...] in der ersten Sitzung ja auch nicht da (war) bzw. erst nach der Hälfte kam. Aber, eben auch vor Erfahrungen wie diesen finde ich es irgendwie relevant, noch mal sozusagen einen Bogen zu schlagen. So denke ich im Moment zumindest» (Sprachmemo 018, 0:12-1:27 min, 10.07.2015).

Durch den Wechsel der Perspektiven erlebte ich hinsichtlich der Gestaltung der letzten Seminarsitzung einen Handlungsdruck auf zwei Ebenen: Als Lehrender hatte ich die Aufgabe, die Präsenzveranstaltung lernförderlich für die Studierenden zu gestalten und als Forscher die Aufgabe, den Rahmen für eine angemessene qualitative und quantitative Veranstaltungsevaluation zu schaffen. Mit dem erprobten Entwurf wurde versucht, dieses Spannungsfeld auszubalancieren (Kap. 7.2.4.1). 


\subsection{Zusammenfassung}

Das vorliegende Kapitel dokumentiert die Ergebnisse der Forschungsphase «Auswertung und Neuperspektivierung» (Kap. 3.1) der ersten Iteration im Sommersemester 2015. Im Rahmen des Kapitels wurden die empirischen Ergebnisse (Kap. 7.4) unter Berücksichtigung des entwickelten Seminarkonzeptes (Kap. 6) diskutiert und ausgewertet. In Anlehnung an die zwei zentralen Ziele des Entwurfs erfolgte die Darstellung mit zwei Schwerpunkten: Metaphern als Artikulations- und Reflexionsanlässe (Kap. 8.2 und Mitgestaltung eines Wikibooks als Erfahrungs- und Reflexionsmöglichkeit (Kap. 8.3).

Hinsichtlich des ersten Schwerpunktes wurde gezeigt, dass die Erstellung und Diskussion von Metaphern im aktuellen Praxisentwurf verschiedene Potenziale für Lern- und Lehrhandlungen eröffnen können. Diese wurden in der Erprobung von Studierenden und Lehrenden (zumindest partiell) genutzt (Kap. 8.2.1, 8.2.2). Zur Identifikation neuer Handlungsspielräume auf mikrodidaktischer Ebene wurden potenzielle Diskussionsthemen formuliert, welche auf Basis der formulierten studentischen Metaphern thematisiert werden können: «Rollenbilder von Lehrenden und Lernenden» (Kap. 8.2.2.1), «Bedeutung von Methoden und Medien für das Lehren und Lernen» (Kap. 8.2.2.2), «Gendergerechte Sprache der Metaphern» (Kap. 8.2.2.3) sowie «Möglichkeiten und Grenzen metaphorischer Vergleiche» (Kap. 8.2.2.4).

Eine Beantwortung der Frage, inwiefern die Mitgestaltung eines öffentlichen Wikibooks neue Erfahrungen zum Lernen mit sowie zum Lernen über Medien ermöglichte, erfolgte hinsichtlich ausgewählter Aspekte. Lerngelegenheiten zum Lernen über Medien bzw. zur Entwicklung einer Fähigkeit zur Teilhabe an partizipativen Medienkulturen eröffneten sich für einen Teil der Studierenden. Dies erfolgte durch Gestaltung eigener Produkte im Web (Kap. 8.3.3) sowie durch die Einblicke in eine partizipative Sharing-Gemeinschaft (Kap. 8.3.4). Zudem wurden durch das Seminar neue Möglichkeiten zur Verwendung digitaler Medien in der Hochschullehre für die Studierenden erfahrbar. Diese Erfahrungen wurden als positive Abwechslung zu bekannten Seminarformen bewertet (Kap. 8.3.1). Zugleich zeigte sich auch, dass das kollaborative Schreiben und Lernen in öffentlichen Wikis für viele Studierende eine Herausforderung darstellte (Kap. 8.3.2)

Die im Rahmen der ersten Iteration vorgestellten Praxisreflexionen (Kap. 7.2), die präsentierten empirischen Ergebnisse (Kap. 7.4) sowie deren Diskussion und Interpretation im Rahmen dieses Kapitels (Kap. 8) markieren den Abschluss des ersten Forschungszyklus. Zugleich können Sie als Übergang zum zweiten Forschungszyklus verstanden werden. So wurde auf Basis der verschiedenen Erkenntnisse eine Modifikation des Konzeptes durchgeführt (Kap. 9), welches im Wintersemester erneut erprobt und wissenschaftlich untersucht wurde (Kap. 10). 


\section{Entwurfsmodifikationen}

\subsection{Methodische Verortung}

Die Überarbeitung bzw. Modifikation des entwickelten Entwurfs zur Durchführung eines Wikibookprojektes als Seminar in der ersten Phase der Lehrerinnen- und Lehrerbildung (Kap. 6) stellt den Gegenstand des aktuellen Kapitels dar. Im Prozessmodell der entwicklungsorientierten Bildungsforschung von Sesink und Reinmann (2015) kann die Entwurfsmodifikation als zweite Iteration der Forschungsphase «Problematisierung und Entwurf» (Kap. 3.1.1) verstanden werden. Auf Basis der Analyse (Kap. 7) sowie der Auswertung (Kap. 8) werden insbesondere auf einer mikrodidaktischen Ebene Veränderungen beschrieben und diskutiert. Ausgangspunkt für die skizzierten Veränderungen ist der erste entwickelte Entwurf zur Durchführung eines Wikibookprojektes (Kap. 6). Für die schematische Darstellung des Prozessmodells wurde entschieden, die zweite Iteration als Spirale darzustellen, um deutlich zu machen, dass der modifizierte Entwurf auf dem ersten Entwurf basiert und zugleich über diesen hinausgeht (Abb. 9.1).

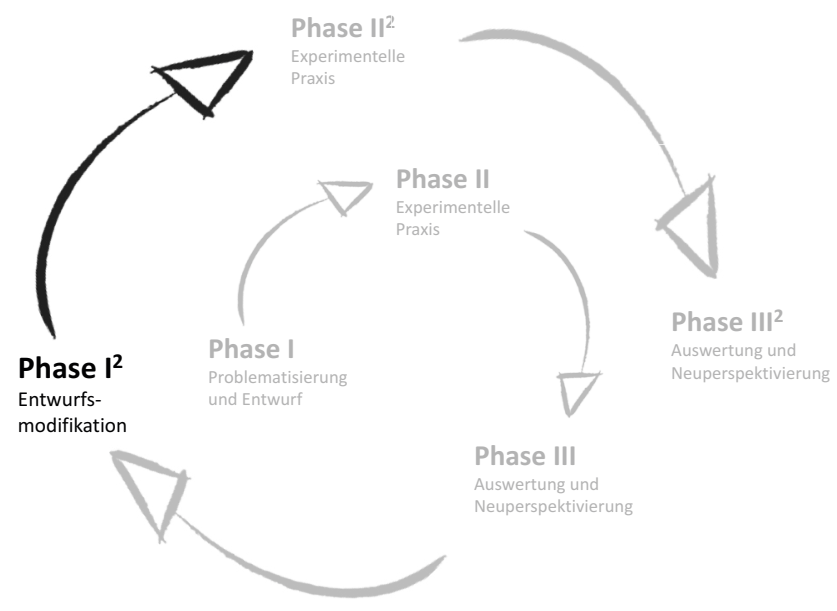

Abb. 9.1: Verortung des Kapitels im Phasenmodell der entwicklungsorientierten Bildungsforschung

Dem Verständnis von Tulodziecki et al. $(2013,227)$ folgend, dient die Darstellung der modifizierten didaktischen Entscheidungen dem Zweck, die «Gestaltung als Bestandteil des Forschungsprozess[es] und der Wissensgenerierung» anzuerkennen (Kap. 2.2.3). Unter Berücksichtigung der Überlegungen des ersten Entwurfs (Kap. 6), den empirischen Ergebnissen (Kap. 7.4) und den sich neu eröffnenden Handlungsspielräumen (Kap. 8) wird für drei exemplarisch ausgewählte Lernsituationen skizziert, wie bei der Modifikation didaktischer Entscheidungen vorgegangen wurde. 
Die ausgewählten und modifizierten didaktischen Entscheidungen lassen sich nach Wildt (2002) auf der hochschuldidaktischen Handlungsebene zur Planung und Gestaltung von «(Lern)Situationen» verorten bzw. nach Kron et al. (2014) auf «mikrosozialer Ebene» beschreiben (Kap. 4.2.3). Darüber hinaus erfolgt eine Modifikation der bisherigen Seminar- und Phasenstruktur, um die organisatorischen und zeitlichen Rahmenbedingungen zur Realisierung der veränderten Lernsituationen zu schaffen (Kap. 9.3). Diese Entscheidungen können nach Wildt (2002) auf der Ebene der «(Lehr) Veranstaltung» verortet werden.

\subsection{Modifikation ausgewählter Lernsituationen}

Die drei exemplarisch ausgewählten Lernsituationen beinhalten erweiterte Aufgabenstellungen zur Visualisierung von Metaphern als neue Reflexionsmöglichkeit (Kap. 9.2.1) und skizzieren die Überlegungen zur Eröffnung von Mitbestimmungsmöglichkeiten hinsichtlich verschiedener Gruppenarbeitsprozesse (Kap. 9.2.2). Mit der Betrachtung des Wikibooks als gestaltbares und offenes Lehr- und Lernmaterial (Kap. 9.2.3) werden abschliessend neue Handlungsspielräume zur Durchführung eines Wikibookprojektes skizziert und Konsequenzen für die Gestaltung weiterer Lernsituationen benannt.

\subsubsection{Visualisierung von Metaphern als neue Reflexionsmöglichkeit}

Ein zentrales Ziel des Seminarkonzeptes ist es, die (Weiter-)Entwicklung der pädagogischen Artikulations- und Reflexionsfähigkeit der Studierenden zu fördern und zu unterstützen (Kap. 6.2.1). Unter Berücksichtigung der Voraussetzungen der Studierenden (Kap. 6.3.1) wurde die Annahme formuliert, dass bestimmte Lernhandlungen für diese (Weiter-)Entwicklung sinnvoll sind (Kap. 6.4.1). Auf Basis der Prozessreflexionen der Erprobung (Kap. 7.2), den Ergebnissen der Analyse (Kap. 7.4.3) sowie der Auswertung (Kap. 8.2) wurde entschieden, die Aufgabenstellungen zur Entwicklung von Metaphern zu modifizieren. Während bis dahin die Artikulationsmöglichkeiten weitgehend auf sprachliche bzw. schriftliche Formen der Metaphernformulierung beschränkt waren, wurden diese um Visualisierungsmöglichkeiten erweitert. In der individuellen und gemeinsamen Visualisierung der formulierten Metaphern wird folgendes Potenzial gesehen:

- In der Analyse der Gruppenmetaphern wurde das Phänomen beschrieben, dass diese auf den (metaphorischen Mustern der) Ad-hoc-Metaphern basieren. Zugleich beinhalten die Gruppenmetaphern weitere (teilweise modifizierte) Elemente (Kap. 8.2.3.1). Dabei wurde auch das Phänomen beschrieben, dass die verschiedenen Aspekte der individuellen Vorstellungen aus der Perspektive der Studierenden «verloren» gegangen sind (Kap. 7.2.2.1). Um Studierenden die 
Gelegenheit zu eröffnen, ihre individuelle Sicht auf die formulierte Gruppenmetapher zum Ausdruck zu bringen, erscheinen individuelle Visualisierungsmöglichkeiten als geeignete Möglichkeit (Kap. 6.4.1.1).

- Die gemeinsame Visualisierung der Gruppenmetaphern bietet einen Anlass für Diskussionen zwischen den Studierenden. In entsprechenden Aushandlungsprozessen wird die Chance gesehen, Anlässe zur Irritation sowie zur Reflexion der eigenen Vorstellungen zu schaffen (Kap. 6.4.1.2).

- In der Gestaltung und Visualisierung von Metaphern wird das Potenzial gesehen, in einem handlungsorientierten Vorgehen Anlässe zur Weiterentwicklung der eigenen Medienkompetenz zu schaffen. In der eigenen Gestaltung und Visualisierung besteht beispielsweise die Möglichkeit, Kompetenzen im Bereich der Auswahl und Nutzung angemessener Bildbearbeitungssoftware zu entwickeln. In den Bereichen der Gestaltung und Veröffentlichung eigener Medienbeiträge (Kap. 6.4.2.1) sowie in der Beurteilung von Bedingungen zur Produktion und Verbreitung öffentlicher Medienbeiträge (Kap. 6.4.2.2) kann die Recherche von Bildern und Animationen unter freien und offenen Lizenzen sinnvolle Lernanlässe schaffen.

Die Realisierung dieser Überlegungen erfolgte in Form von modifizierten Aufgabenstellungen im Rahmen des Kick-off-Events der ersten Seminarphase (Kap. 6.6.1.1). Folgende Gestaltungsentscheidungen wurden getroffen, um Anlässe zur Artikulation eigener Vorstellungen zu schaffen, (Kap. 6.4.1.1), eine Auseinandersetzung mit alternativen Sprachfiguren und Perspektiven anzuregen (Kap. 6.4.1.2) sowie die Entwicklung der eigenen Medienkompetenz zu unterstützen (Kap. 6.4.2.1, 6.4.2.2).

- In der zweiten Phase des Kick-off-Termins wurden Studierende mit der Aufgabe konfrontiert, in Form einer modifizierten Think-Pair-Share-Methode eine konsensfähige Gruppenmetapher zu entwickeln (Kap. 6.6.1.1, Anhang D.5, D.6). In Erweiterung dieser Aufgabenstellung wurden die studentischen Arbeitsgruppen mündlich dazu ermuntert, ihre entwickelte Metapher auch visuell aufzubereiten, z. B. in Form einer eigenen Darstellung oder eines recherchierten Bildes. Zur eigenen Visualisierung wurden den Studierenden Flipcharts sowie ein interaktives Whiteboard zur Verfügung gestellt. Zur Recherche von angemessenen Bildern hatten die Studierenden Zugriff auf Laptops. Die damit erstellten und recherchierten Visualisierungen der vorläufigen Gruppenmetaphern dienten im Rahmen der gegenseitigen Präsentation und Diskussion als Orientierungsmöglichkeiten für die weiteren Arbeitsgruppen.

- Die dritte Phase des Kick-off-Events zur «digitalen Produktion» wurde um eine individuelle Visualisierungsaufgabe erweitert. Neben der Erstellung einer Videobotschaft für Studierende der «Escola Superior de Educação de Viseu» wurde die folgende Aufgabenstellung formuliert: «Recherchieren/Erstellen Sie Visual 
Notes zur Veranschaulichung Ihrer Metapher (jeder in Form eines ‘Fotoalbums) im Moodle-Forum Deadline: Heute!)» (Anhang D.34). Die erstellten und recherchierten Visualisierungen präsentieren die jeweils individuelle Perspektive auf die gemeinsam erstellte Gruppenmetapher und die Möglichkeit für Reflexionen in der dritten Seminarphase.

Mit der Modifikation und Erweiterung der zwei skizzierten Aufgabenstellungen im Rahmen des konkreten Seminarkonzeptes wird die Annahme verbunden, die (Weiter-)Entwicklung der pädagogischen Artikulations- und Reflexionsfähigkeit der Studierenden auf neue Weise zu fördern und zu unterstützen (Kap. 6.2.1).

\subsubsection{Modus der Gruppenbildung als Mitgestaltungsmöglichkeit}

Zur Anregung der als sinnvoll erachteten Lernhandlungen (Kap. 6.4) wurde die Gestaltung von Kooperations- und Kollaborationsanlässen der Studierenden als relevante Lehrhandlung skizziert (Kap. 6.5.2). Im Rahmen des vorgestellten BlendedLearning-Konzeptes wurden die verschiedenen didaktischen Gestaltungsentscheidungen für die unterschiedlichen Seminarphasen konkretisiert (Kap. 6.6.1). Die in den Interviews (Kap.7.4.1.3) und den Gruppengesprächen (Kap. 7.4.2.2.5) zum Ausdruck gebrachte Kritik an der Aktivität der Gruppenmitglieder sowie zur Gruppenbildung wurden als Indikatoren dafür gesehen, das Konzept der wechselnden Gruppen sowie der Gruppenbildung zu modifizieren (Kap. 6.6.1.2).

Im Rahmen der ersten Erprobung wurden Studierende über das geplante methodische Vorgehen in der zweiten Phase informiert. Dieses Vorgehen kann nach Mayrberger (2013b, 169) als «Einbeziehung» der Studierenden bezeichnet werden. Darunter versteht Mayrberger (2013b, 169) die Vorbereitung einer Lernumgebung durch die Lehrenden sowie die angemessene Information der Lernenden, dass diese «verstehen worum es geht und wissen, was das Vorhaben bewirken soll» (ebd.). Dieses Vorgehen ist nach Mayrberger (2013b) jedoch nur als Vorstufe von partizipativen Lernen in Bildungsinstitutionen zu bewerten. Für die Modifikation des Entwurfs wurde versucht, die formulierten Lehrhandlungen zur Eröffnung von Partizipationsmöglichkeiten und Erfahrungsorientierung stärker zu berücksichtigen (Kap. 6.5.3). Die Wahl für ein partizipatives Vorgehen, bei dem die Studierenden in die Entscheidung zum weiteren methodischen Vorgehen im Seminar einbezogen werden, entspricht zudem der Bedeutungsdimension einer Entwicklung «als reflexiver Prozess», wie es Sesink und Reinmann $(2015,2)$ skizzieren (Kap. 2.3.3.1).

Die konkrete Realisierung einer gemeinsamen Seminarplanung mit den Studierenden wurde für den Beginn der zweiten Projektphase geplant. Zur Information der Studierenden wurden Folien mit ausgewählten Erkenntnissen der ersten Erprobung (als Anlass zur Diskussion zum weiteren Vorgehen) sowie mit den sich eröffnenden 
Mitgestaltungsmöglichkeiten vorbereitet (Abb. 9.2). Zur Diskussion gestellt wurden drei Aspekte: (1.) Möglichkeiten zur weiteren Gruppenzusammenarbeit, (2.) die Wahl der thematischen Schwerpunkte sowie (3.) die Gestaltung der gemeinsamen Präsenzzeit im Rahmen der zweiten Seminarphase.

Die Präsentation der Folien sowie einer zugehörigen Information für die Studierenden diente dem Ziel, sie angemessen über die benannten Entscheidungsfragen aufzuklären. Dies wurde als Voraussetzung gesehen, um diese Möglichkeiten im Folgenden diskutieren zu können. Im Rahmen der Präsentation wurden sinnvolle methodische Vorgehensweisen begründet und erste Handlungsalternativen skizziert. Auf dieser Basis sollte eine Diskussion mit Studierenden angeregt werden, welche zugleich offen für weitere Vorschläge und Ideen ist. Zum Abschluss war geplant, über die weitere Gruppenzusammenarbeit sowie über die Gestaltung der gemeinsamen Präsenzzeit im Rahmen des Treffens abzustimmen. Für die Wahl eines thematischen Schwerpunktes wurde den Studierenden die Möglichkeit eröffnet, an einer OnlineUmfrage teilzunehmen.
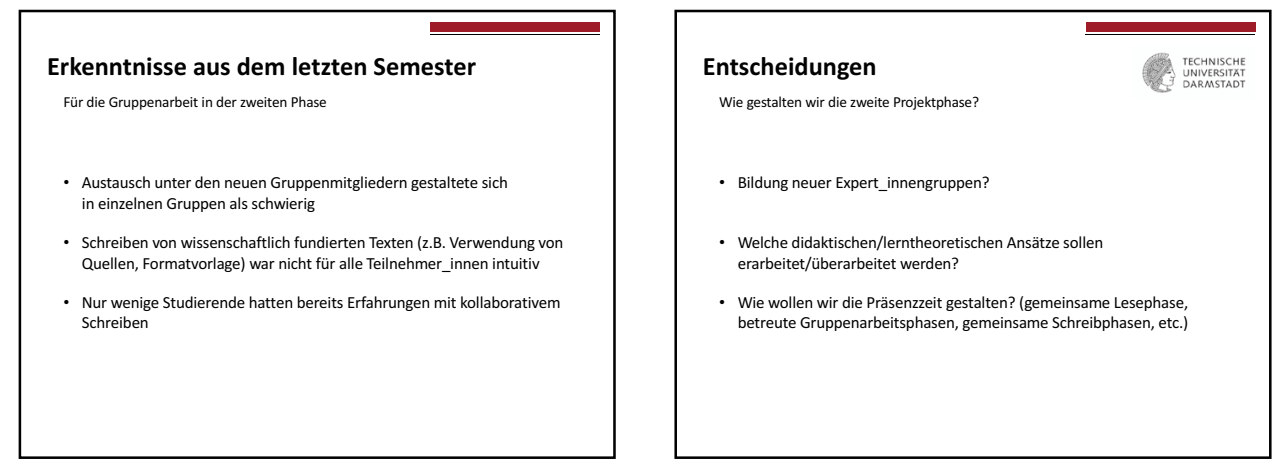

Abb. 9.2: Vorbereitete Folien für die Diskussion mit den Studierenden (eigene Darstellung).

Mit der Modifikation und Erweiterung des Modus der Gruppenbildung als Mitgestaltungsmöglichkeit wurde das Ziel verfolgt, der Kritik der Studierenden gerecht zu werden und potenzielle Lernhandlungen nicht durch organisatorische Probleme zu verhindern. Zudem wurde in dem modifizierten Entwurf die Chance gesehen, die Lehrhandlungen zur Eröffnung von Partizipationsmöglichkeiten besser realisieren zu können und Entwicklungsmöglichkeiten hinsichtlich aller drei Bedeutungsdimensionen nach Sesink $(2015,2)$ zu eröffnen (Kap. 2.3.3.1).

\subsubsection{Wikibook als offenes und veränderbares Lehr- und Lernmaterial}

Der erste Entwurf zur Integration des Wikibooks basierte auf begründeten Annahmen über sinnvolle Lern- und Lehrhandlungen (Kap. 6.4, 6.5). Die Entscheidung für Wikibooks sowie für die Durchführung eines Publikationsprojektes orientierte sich $u$. a. 
an den empirischen Projekten von Bonk et al. (2009) und Xiao und Lucking (2008) (Kap. 6.6.2). Auf Basis der Prozessreflexionen (Kap. 7.2) und den empirischen Ergebnissen (Kap. 7.4.1, 7.4.2, 7.4.4) wurde zum einen gezeigt, dass verschiedene Ziele mit dem entwickelten Entwurf erreicht werden konnten (Kap. 8.3). Zum anderen konnten neue Handlungsspielräume zur Nutzung des Wikibooks im Rahmen des Seminars identifiziert werden (Kap. 8.3.5), deren praktische Konzeption im Folgenden konkretisiert wird.

In der Auftaktveranstaltung erfolgte eine Modifikation der vierten Phase «digitale Produktion». Neben der Erstellung einer Videobotschaft sowie der Recherche von Visual Notes (Kap. 9.2.1) erfolgte eine erste Auseinandersetzung mit Wikibooks, um eine instrumentelle Nutzung des Wikis sowie Wiki-Editors im Rahmen der Präsenztermine zu fördern. Die Studierenden wurden mit den folgenden Aufgabenstellungen konfrontiert:

- Als Einstieg in die Gestaltung des Wikibooks sowie zur Vorbereitung der Teilhabe an einer Sharing-Community wurden Studierenden folgende Aufgabe gestellt: «Erstellen Sie sich einen Account auf wikibooks.org» (Anhang D.35). Dafür mussten die Studierenden sich für einen Accountnamen sowie ein Passwort entscheiden. Weitere Angaben (z. B. Angabe der E-Mail-Adresse) waren optional. Bei der Vorstellung der Aufgabe wurde den Studierenden in der Präsenz gesagt, dass Pseudonyme möglich sind und es ihre Entscheidung sei, unter welchem Namen ihre Beiträge auf Wikibooks sichtbar sein sollen.

- Daran anschliessend bekamen die Studierenden die Aufgabenstellung zur Formulierung ihrer entwickelten Gruppenmetapher: «Schreiben Sie als Gruppe Ihren Beitrag für das Wikibook. Ziel des Beitrages ist es, Ihre Vorstellung von «Lehren und Lernen> als Metapher auszudrücken sowie die Grenzen und Probleme der Metapher deutlich zu machen. (Deadline: Bis zum 28. Oktober auf Wikibooks)» (Anhang D.35). In Anknüpfung an die erste Erprobung (Kap. 7.2.3.1) wurde zudem der folgende Gestaltungshinweis gegeben: «Der Beitrag muss eine Tabelle beinhalten (siehe Flipchart) und sollte 1.000 Wörter nicht überschreiten». Diese Aufgabe wurde im Rahmen von individuellen Gruppenberatungen erläutert, dabei wurden die wesentlichen Funktionen des Wiki-Editors zur Erstellung und Bearbeitung von Wikiseiten mündlich mitgeteilt sowie auf Unterstützungs- und Hilfsmaterialien verwiesen.

Zur Modifikation ausgewählter Präsenztermine wurden Lernsituationen entwickelt, bei denen die Wikibeiträge als offene und veränderbare Einführungs- und Orientierungstexte verwendet werden konnten. Die Auseinandersetzung mit Texten des Wikibooks sollte zum einen eine fachliche Orientierung bieten und zum anderen die Prozessualität und Gestaltbarkeit von Wikitexten erfahrbar werden lassen. Zum Abschluss der ersten Seminarphase erfolgte eine Präsenzveranstaltung, welche sich der 
Erfassung des aktuellen Projektstatus, insbesondere der Diskussion, Kritik und Überarbeitung der Beiträge im Wikibook widmen sollte. Im Rahmen des Seminars wurde geplant, die Studierenden mit drei Aufgabenstellungen zu konfrontieren:

- Lesen der Einleitung (Anhang E.2) und des Einführungstextes zum «Alltagswissen und Metaphern» (Anhang E.3) unter den zwei Fragestellungen: Inwiefern erscheinen die Formulierung nachvollziehbar? Wo besteht Diskussions-/Überarbeitungsbedarf? (Anhang D.36).

- Kriteriengeleitete Betrachtung der Gruppenmetaphern des ersten Semesters (Anhang E.3) sowie Diskussion der zwei Fragestellungen: «Inwiefern erscheinen die Formulierung nachvollziehbar? Wo besteht Diskussions-/Überarbeitungsbedarf? (Anhang D.37).

- Lesen und Diskussion des Feedbacks zu den selbst erstellten Gruppenmetaphern sowie Überarbeitung der eigenen Beiträge (Anhang D.37).

Zu Beginn der zweiten Seminarphase wurde sich ähnliches Vorgehen gewählt, um eine Auseinandersetzung mit den Texten des Wikibooks zu initiieren. Das Ziel war erneut, dass die Studierenden die zugehörigen Texte im Wiki lesen und hinsichtlich vorgegebener Fragestellungen diskutieren. Zur Diskussion des Kapitels «Erziehungswissenschaftliche Denkwerkzeuge» im Wikibook (Anhang E.59 - E.62) wurden die drei folgenden Fragen gestellt:

- Inwiefern wird die Differenz zwischen Alltagswissen und erziehungswissenschaftlichen Denkwerkzeugen nachvollziehbar?

- Inwiefern ist die Relevanz einer Auseinandersetzung mit erziehungswissenschaftlichen Denkwerkzeugen nachvollziehbar?

- Was wird unter den Begriffen (1) Gegenstandsbereich, (2) analytische und (3) normative Dimension eines Modells verstanden? (Anhang D.43)

Die Fragestellungen wurden mit dem Ziel formuliert, Anlässe für Lernhandlungen zur Entwicklung der pädagogischen Artikulations- und Reflexionsfähigkeit zu eröffnen. In Anlehnung an Terhart (2009) bietet der formulierte Text zur Auseinandersetzung mit einer pädagogischen Perspektive u. a. einen Orientierungsrahmen zur Differenzierung unterschiedlicher Wissensformen über pädagogische Zusammenhänge. Mit Bezug auf Terhart (2009) und Peterssen (2001) werden Argumente formuliert, um die Relevanz einer pädagogischen Perspektive für angehende Lehrerinnen und Lehrern zu begründen (Kap. 6.4.1.3). Zudem werden konkrete implizite und explizite Merkmale didaktischer Modelle formuliert. In der Diskussion der Darstellung sowie der Nachvollziehbarkeit der formulierten Argumente eröffnet sich neben der inhaltlichen Auseinandersetzung auch die Möglichkeit, über die Prinzipien eines Wikibeitrages zu sprechen. Über konkrete Änderungsvorschläge der Studierenden und der entsprechenden Bearbeitung der Beiträge kann der Prozesscharakter und die Veränderbarkeit in der Situation zugänglich gemacht werden. 
Neben der Diskussion der Kapiteleinleitungen eröffneten die studentischen Produkte der vorherigen Erprobung interessante Materialien. Interessant sind die studentischen Wikibeiträge zum einen, weil sie verschiedene didaktische Modelle und lerntheoretische Überlegungen in der vorgeschlagenen Struktur der Kapiteleinleitung präsentierten. Interessant sind diese Beiträge zum anderen, weil sich die Qualität der Beiträge unterscheidet. Für die jeweils neuen Teilnehmerinnen und Teilnehmer stellt sich die Herausforderung, die Qualität der unterschiedlichen Beiträge zu bewerten. Zur Anregung von Diskussionen wurden die Studierenden mit folgenden Fragestellungen konfrontiert:

- Welche Beiträge erscheinen euch ${ }^{257}$ informativ? Welche Beiträge erscheinen euch qualitativ angemessen?

- Wie kann die Qualität der bisherigen Beiträge eingeschätzt werden? (Anhang D.43)

Vor dem Hintergrund der Lernvoraussetzungen der Studierenden wurde die Annahme vertreten (Kap. 6.3.1), dass diese Einschätzung kaum auf inhaltlichem Vorwissen aufbauen kann. In dieser Überforderungssituation wurde jedoch die Chance gesehen, die Studierenden einerseits für die inhaltlichen Zielstellungen zu sensibilisieren und zum anderen Nachfragen anzuregen. Ferner eröffneten die studentischen Beiträge einen Einblick in mögliche Themen zur Erarbeitung bzw. Überarbeitung im Rahmen der Gruppenphase. Zuletzt wurde in der Diskussion über die Qualität der studentischen Beiträge die Möglichkeit gesehen, über formale Gestaltungsprinzipien wissenschaftlich erarbeiteter Texte im Rahmen eines Wikibooks zu sprechen. In diesem Sinne bot die gemeinsame Diskussion und Überarbeitung von Wikibeiträgen auch die Möglichkeit, Lernhandlungen zur (Weiter)Entwicklung der Fähigkeit zur Teilhabe an einer partizipativen Kultur anzuregen (Kap. 6.4.2).

\subsection{Modifikation der Prozessplanung}

Zur praktischen Realisierung und Erprobung der formulierten didaktischen Überlegungen (Kap. 6.2, 6.3, 6.4, 6.5) wurde im Rahmen des ersten Entwurfs ein BlendedLearning-Konzept vorgestellt (Kap. 6.6.1). Dieses basierte auf den zuvor formulierten Annahmen und beinhaltete Konkretisierungen zur Medienwahl, zu den Inhalten sowie zur Prozessplanung und dem Verhältnis von Präsenzterminen zu (digitalen) Schreibphasen. Die positiven Rückmeldungen im Rahmen der Veranstaltungsevaluation (Kap. 7.4.2.1), in den Gruppengesprächen (Kap. 7.4.2.2) sowie den Einzelinterviews (Kap. 7.4.1) wurden als Indikator gewertet, die grundlegende Projektidee sowie die Strukturierung der Seminarphasen fortzuführen. Zugleich wurden die Erfahrungen im Verlauf des Seminars (Kap. 7.2), der studentische Umgang mit den

257 Im Verlauf der Seminare veränderte sich die gewählte Anrede der Studierenden. Für Aufgabenstellungen in Präsenzterminen wurde überwiegend das Anredepronomen «ihr» (statt «sie») verwendet. 
formulierten Rückmeldungen, die kritischen Hinweise zur Gruppenorganisation sowie das ambivalent bewertete Verhältnis von freien Lernphasen zu vorstrukturierten Präsenzzeiten (Kap. 7.4.2.2.6) als Anlass verstanden, das Verhältnis von Präsenzveranstaltungen und freien Schreibphasen neu zu balancieren.

Bei der Modifikation der ersten Prozessplanung wurde die gewünschte Auseinandersetzung mit formulierten Rückmeldungen als Präsenzsitzung geplant und nicht mehr den Studierenden als Selbstorganisation überlassen. Diese Entscheidung erfolgte aus zwei Gründen: Die Gestaltung einer Präsenzsitzung zur Besprechung von Rückmeldungen eröffnete die Möglichkeit, mündlich Rückfragen zu Unklarheiten zu formulieren ohne dies über das Moodle-Forum verschriftlichen zu müssen. Selbst wenn in der Verschriftlichung der eigenen Gedanken und Fragen eine Reflexionsmöglichkeit gesehen wird, musste bei Betrachtung der ersten Erprobung markiert werden, dass Studierende diese Möglichkeiten weitgehend nicht nutzten (Kap. 7.2.2.2, 7.2.3.2, 7.2.4.2). Ein weiteres Argument für die Durchführung einer Präsenzsitzung waren die Interviewaussagen von Studierenden, aus denen hervorging, dass sie die Rückmeldungen zwar wahrnahmen und als sinnvoll erachteten, eine Überarbeitung der eigenen Schreibprodukte dennoch häufig ausblieb. In den Interviews und Gruppengesprächen wurde dies u. a. auf Probleme bei der Organisation der Arbeitsgruppen zurückgeführt (Kap. 7.4.1.3, 7.4.2.2.5) Diese Problematik wurde auch bei der Prozessreflexion der ersten Erprobung (Kap. 7.2.4.1) markiert und führte zu einer Planungskorrektur hinsichtlich der Zeitplanung des ersten Entwurfs (Abb. 7.8). Die Durchführung einer Präsenzsitzung schien vor diesem Hintergrund hilfreich zu sein, um den Arbeitsgruppen einen Anlass für ein gemeinsames Treffen zu geben, bei dem sie - neben der Diskussion der Rückmeldungen - mit der Überarbeitung der eigenen Produkte beginnen konnten.

Mit der Modifikation des Phasenkonzeptes zur Balancierung des Verhältnisses von vorbereiteten Präsenzsitzungen und selbst organisierten Schreibphasen (Abb. 9.3) wurde insbesondere das Ziel verfolgt, einen geeigneten zeitlichen Rahmen für die modifizierten Lernsituationen zu schaffen. So eröffneten die weiteren Präsenzveranstaltungen die Möglichkeit, die individuellen Fragen zu den Rückmeldungen der potenziell abgeschlossenen Seminarphase vom Beginn der jeweils neuen Seminarphase zu trennen. Zudem eröffneten sich in den Präsenzveranstaltungen zu Beginn der zweiten und dritten Projektphase jeweils neue zeitliche Spielräume für die modifizierte Integration des Wikibooks (Kap. 9.2.3) und die gemeinsame Seminarplanung mit den Studierenden (Kap. 9.2.2). Zugleich muss die Prozessplanung für die zweite und dritte Seminarphase als vorläufig bezeichnet werden, da sie gemeinsam mit den Studierenden überarbeitet werden kann (Kap. 9.2.2). 


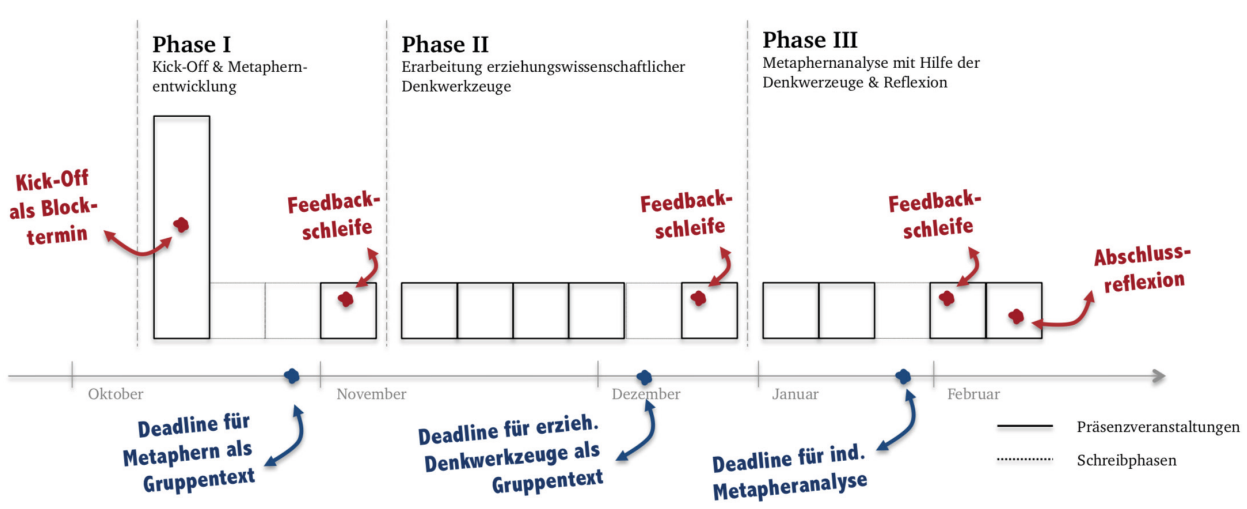

Abb. 9.3: Visualisierung der Präsenzveranstaltungen und Schreibphasen (eigene Darstellung).

\subsection{Zusammenfassung}

Das vorliegende Kapitel skizzierte die Modifikation didaktischer Gestaltungsentscheidungen auf Basis der bisherigen Erkenntnisse (Kap. 6, 7.2, 8). Die didaktischen Überlegungen erfolgten an exemplarisch ausgewählten Bereichen, welche sich überwiegend auf der hochschuldidaktischen Handlungsebene der Lernsituationen verorten lassen. Im Rahmen des gesamten entwicklungsorientierten Bildungsforschungsprojektes stellte das Kapitel einen Bestandteil des Übergangs zwischen dem ersten und dem zweiten Projektdurchlauf dar.

Nach der methodischen Verortung des Kapitels (Kap. 9.1) widmeten sich die vorgestellten Überlegungen insbesondere der Modifikation ausgewählter Lernsituationen (Kap. 9.2). Dabei wurde skizziert, wie neue Aufgabenstellungen zur Visualisierung von Metaphern verwendet werden können, um neue Reflexionsmöglichkeit zu eröffnen (Kap. 9.2.1). Mit diesen Aufgabenstellungen wurde das Ziel verfolgt, Lernhandlungen zur (Weiter-)Entwicklung der pädagogischen Artikulations- und Reflexionsfähigkeit anzuregen. Zur Förderung der Fähigkeit zur Teilhabe an einer partizipativen Medienkultur wurde auch die Integration des Wikibooks überarbeitet und stärker als zuvor als offenes Lehr- und Lernmaterial konzipiert (Kap. 9.2.3). In der vorgestellten Planung wurde die Chance gesehen, die (Weiter-)Entwicklung der eigenen Medienkompetenz der Studierenden anzuregen sowie zugleich Anlässe zur (Weiter-)Entwicklung der pädagogischen Artikulations- und Reflexionsfähigkeit zu schaffen. Ebenfalls dargestellt wurde die Überarbeitung des Modus zur Gruppenbildung im Rahmen der zweiten Projektphase (Kap. 9.2.2). Dies erfolgte insbesondere mit dem Ziel, die im Rahmen der ersten Erprobung problematisierten Aspekte der Gruppenzusammenarbeit zu verbessern bzw. unter der Mitgestaltung der Studierenden zu organisieren. 
Abschliessend wurde die Modifikation der Seminarstruktur bzw. der Prozessplanung skizziert, um die benannten Lernsituationen angemessen realisieren zu können (Kap. 9.3). Diese Anpassung beinhaltete u. a. die Reduktion selbst organisierter Schreibzeiten, um Feedbacksitzungen als Präsenzveranstaltungen durchführen zu können. Zugleich muss das modifizierte Konzept für verschiedene Lernsituationen als vorläufig betrachtet werden, da Studierende insbesondere in der zweiten Seminarphase mehr Mitbestimmungsmöglichkeiten erhalten sollen.

Der modifizierte Entwurf kann mit den Worten von Sesink und Reinmann (2015, 75) als zweite Version einer «in der Vorstellung vorweg genommene «bessere» Praxis» beschrieben werden. Im Rahmen des vorliegenden Projektes wurde auch diese Vorstellung erprobt und wissenschaftlich untersucht. Diese Erprobung wird - analog zum Vorgehen in der ersten Iteration - im folgenden Kapitel «Experimentelle Praxis» (Kap. 10) aus der Perspektive des Lehrenden reflektiert und mithilfe wissenschaftlicher Methoden empirisch analysiert.

\section{Literatur}

Abels, Simone. 2011. LehrerInnen als «Reflective Practitioner». Reflexionskompetenz für einen demokratieförderlichen Naturwissenschaftsunterricht. Wiesbaden: VS Verlag für Sozialwissenschaften.

Bonk, Curtis J., Mimi Miyoung Lee, Nari Kim, und Meng-Fen Grace Lin. 2009. "The tensions of transformation in three cross-institutional wikibook projects». The Internet and Higher Education 12 (3-4): 126-35. http://dx.doi.org/10.1016/j.iheduc.2009.04.002.

Flick, Uwe. 2011. Triangulation: eine Einführung. 3., akt. Aufl. Bd. 12. Qualitative Sozialforschung. Wiesbaden: VS Verlag für Sozialwissenschaften.

Gansen, Peter. 2010. Metaphorisches Denken von Kindern: theoretische und empirische Studien zu einer pädagogischen Metaphorologie. Bd. 56. Erziehung Schule Gesellschaft. Würzburg: ERGON Verlag.

Grell, Petra. 2006. Forschende Lernwerkstatt. Bd. 472. Internationale Hochschulschriften. Münster [u. a.]: Waxmann.

Heinmann, Paul. 1962. «Didaktik als Theorie und Lehre». Die Deutsche Schule 54 (9): 407-427.

Holzkamp, Klaus. 1995. Lernen: subjektwissenschaftliche Grundlegung. Studienausg. Frankfurt/Main [u. a.]: Campus Verlag.

Iske, Stefan, und Winfried Marotzki. 2010. «Wikis: Reflexivität, Prozessualität und Partizipation». In Medienbildung in neuen Kulturräumen. Die deutschprachige und britische Diskussion, herausgegeben von Ben Bachmair, 1. Aufl., 141-151. Wiesbaden: VS Verlag für Sozialwissenschaften. https://doi.org/10.1007/978-3-531-92133-4_10. 
Kardorff, Ernst von. 2012. "Qualitative Evaluationsforschung». In Qualitative Forschung: ein Handbuch, herausgegeben von Uwe Flick, Ernst von Kardorff, und Ines Steinke, Orig.Ausg., 9. Aufl. Bd. 55628 : Rowohlts Enzyklopädie. rororo. Reinbek bei Hamburg: RowohltTaschenbuch-Verl.

Klafki, Wolfgang. 1997. «Die bildungstheoretische Didaktik im Rahmen kritisch-konstruktiver Erziehungswissenschaft». In Didaktische Theorien, herausgegeben von Herbert Gudjons und Rainer Winkel, 9. Auflage, 13-34. Hamburg: Bergmann und Helbig.

Klingberg, Lothar. 1990. Lehrende und Lernende im Unterricht. Berlin: Volk und Wissen Verlag.

Kron, Friedrich W., Jutta Strandop, und Jürgens Eiko. 2014. Grundwissen Didaktik. 6. Auflage. München, Basel: Reinhardt.

Mayrberger, Kerstin. 2013. "Partizipatives Lernen mit dem Social Web in der Schule». In Organisation und Partizipation: Beiträge der Kommission Organisationspädagogik, herausgegeben von Susanne Maria Weber, Michael Göhlich, Andreas Schröer, Claudia Fahrenwald, und Hildegard Macha, 167-175. Wiesbaden: Springer Fachmedien Wiesbaden. https://doi. org/10.1007/978-3-658-00450-7_14.

Peterssen, Wilhelm. 2001. Lehrbuch Allgemeine Didaktik. Oldenbourg Wissenschaftsverlag $\mathrm{GmbH}$.

Schmidt, Jan-Hinrik, und Monika Taddicken, Hrsg. 2017. Handbuch Soziale Medien. Springer Reference Sozialwissenschaften. Wiesbaden: Springer VS.

Sesink, Werner. 2015. Entwicklungsorientierte Bildungsforschung. Plädoyer für einen «dritten Weg» in pädagogischer Forschung. Eine Textsammlung. http://www.sesink.de/wordpress/ wp-content/uploads/2015/11/Entwicklungsorientierte-Bildungsforschung_Sesink_2015. pdf.

Sesink, Werner, und Gabi Reinmann. 2015. «Umrisse eines Strukturmodells für entwicklungsorientierte bildungswissenschaftliche Forschung». In Entwicklungsorientierte Bildungsforschung. Plädoyer für einen ‘dritten Weg) in pädagogischer Forschung. Eine Textsammlung, herausgegeben von Werner Sesink, 69-83. http://www.sesink.de/wordpress/wp-content/ uploads/2015/11/Entwicklungsorientierte-Bildungsforschung_Sesink_2015.pdf.

Steinke, Ines. 2012. "Gütekriterien qualitativer Forschung». In Qualitative Forschung: ein Handbuch, herausgegeben von Uwe Flick, Ernst von Kardorff, und Ines Steinke, 319-331. Reinbek bei Hamburg: Rowohlt-Taschenbuch-Verl.

Terhart, Ewald. 2009. Didaktik : eine Einführung. Stuttgart: Reclam.

TU Darmstadt. 2017. Geschlechtergerecht formulieren. Empfehlungen und Tipps. https://www. tu-darmstadt.de/media/frauenbeauftragte/relaunch/pdf_10/zentrale_dokumente/Geschlechtergerecht_formulieren_Flyer_Druckversion.pdf.

Tulodziecki, Gerhard, Silke Grafe, und Bardo Herzig. 2013. Gestaltungsorientierte Bildungsforschung und Didaktik: Theorie - Empirie - Praxis. Bad Heilbrunn: Klinkhardt.

Universität zu Köln. 2017. ÜberzeuGENDERe Sprache. Leitfaden für eine geschlechtersensible und inklusive Sprache. http://gedim.uni-koeln.de/sites/genderqm/user_upload/Leitfaden_geschlechtersensible_Sprache_5.Auflage_2017.pdf. 
Vervecken, Dries, und Bettina Hannover. 2015. «Yes I Can!» Social Psychology 46 (2): 76-92. https://doi.org/10.1027/1864-9335/a000229.

Vervecken, Dries, Bettina Hannover, und Ilka Wolter. 2013. «Changing (S)expectations: How gender fair job descriptions impact children's perceptions and interest regarding traditionally male occupations». Journal of Vocational Behavior 82 (3): 208-20. https://doi. org/10.1016/j.jvb.2013.01.008.

Wildt, Johannes. 2002. «Ein hochschuldidaktischer Blick auf Lehren und Lernen». In Neues Handbuch Hochschullehre, herausgegeben von Brigitte Berendt, Hans-Peter Voss, und Johannes Wildt, 1-10. Bonn: Raabe-Verlag.

Xiao, Yun, und Robert Lucking. 2008. «The impact of two types of peer assessment on students' performance and satisfaction within a Wiki environment». The Internet and Higher Education 11 (3-4): 186-193. https://doi.org/10.1016/j.iheduc.2008.06.005. 